\title{
Inertia-Free Spacecraft Attitude Control with Control Moment Gyroscope Actuation
}

\author{
Kshitij Agarwal, Avishai Weiss; Ilya Kolmanovsky; \\ and Dennis S. Bernstein ${ }^{\S}$ \\ Department of Aerospace Engineering, The University of Michigan, Ann Arbor, MI 48109-2140
}

\begin{abstract}
We extend the inertia-free continuous control law for spacecraft attitude tracking derived in prior work to the case of three single-axis control moment gyroscopes with spherical gyro wheels. These CMGs are assumed to be mounted in a known and linearly independent configuration with an arbitrary and unknown orientation relative to the spacecraft principal axes. We demonstrate the performance of the modified control laws for rest-to-rest, motionto-rest and spin maneuvers without the need for a seperate steering algorithm.
\end{abstract}

\section{Introduction}

In spacecraft applications it is often expensive to determine the mass properties with a high degree of accuracy. To alleviate this requirement, the control algorithms given in refs. ${ }^{1-3}$ require no prior modeling of the mass distribution. These algorithms incorporate internal states that can be viewed as estimates of the moments and products of inertia; however, these estimates need not converge to the true values, and in fact do not converge to the true values except in cases of sufficiently persistent motion.

The results of ref. ${ }^{1}$ are based on rotation matrices ${ }^{4}$ as an alternative to quaternions as used in refs. ${ }^{3,5,6}$ Quaternions provide a double cover of the rotation group $\mathrm{SO}(3)$, and thus, when used as the basis of a continuous control algorithm, cause unwinding, that is, unnecessary rotation away from and then back to the desired physical attitude. ${ }^{7}$ To avoid unwinding while using quaternions, it is thus necessary to resort to discontinuous control algorithms, which introduces the possibility of chatter due to noise as well as mathematical complications. ${ }^{8-10}$ On the other hand, rotation matrices allow for continuous control laws but introduce multiple equilibria. However, the equilibria that do not represent the desired equilibrium are saddle points of the closed-loop system, and thus the attitude of the spacecraft converges almost globally to the desired equilibrium.

The inertia-free control law developed in ref. ${ }^{1}$ was extended in ref. ${ }^{2}$ to the case of reaction wheel actuation. The goal of the present paper is to further extend the control law to include control moment gyroscope (CMG) actuation. We derive coordinate-free equations of motion for a satellite with three singleaxis spherical CMGs and specialize them to the case where the gyro wheels rotate with a fixed angular velocity and the gimbals are aligned orthogonally with the spacecraft bus. We derive the inertia-free control law for such actuation, and we demonstrate the performance of the control law for rest-to-rest, motion-to-rest and spin maneuvers.

The traditional approach to controlling spacecraft with CMG's is to implement a controller that specifies a torque input, and then command the gimbal angular velocities in order to synthesize the requested torque using a suitable steering algorithm. ${ }^{11}$ However, the gimbal commands must be chosen to avoid singularities that can arise from the alignment of the gimbals. ${ }^{12-16}$ An experiment devoted to singularity avoidance is described in ref. ${ }^{17}$ The singularity problem can be avoided by implementing CMG's with variable gyro wheel speed. $^{18}$

In the present paper we consider fixed speed CMG's, where the adaptive controller directly commands the gimbal angular velocities. This approach avoids the need to separately synthesize gimbal angular velocities

\footnotetext{
${ }^{*}$ Graduate Student, Aerospace Engineering Department

$\dagger$ Graduate Student, Aerospace Engineering Department

$\ddagger$ Professor, Aerospace Engineering Department

$\S$ Professor, Aerospace Engineering Department
} 
from a requested torque. We demonstrate the adaptive controller for rest to rest, motion to rest and spin maneuvers, where singularity avoidance is also demonstrated.

\section{Spacecraft Model with CMGs}

In this section we derive the equations of motion for a spacecraft with CMGs, while highlighting the underlying assumptions on CMG geometry, inertia, and attachment to the bus. Throughout the paper $\vec{r}_{\mathrm{q} / \mathrm{p}}$ denotes the position of point $\mathrm{q}$ relative to point $\mathrm{p}, \vec{v}_{\mathrm{q} / \mathrm{p} / \mathrm{X}}$ denotes the velocity of point $\mathrm{q}$ relative to point $\mathrm{p}$ with respect to frame $\mathrm{F}_{\mathrm{X}}$, and $\vec{\omega}_{\mathrm{Y} / \mathrm{X}}$ denotes the angular velocity of frame $\mathrm{F}_{\mathrm{Y}}$ relative to frame $\mathrm{F}_{\mathrm{X}}$. All frames are orthogonal and right handed.

Def. 1. Let $F_{X}$ be a frame, let $\mathcal{B}$ be a collection of rigid bodies $\mathcal{B}_{1}, \ldots, \mathcal{B}_{l}$, and let $\mathrm{p}$ be a point. Then, the angular momentum of $\mathcal{B}$ relative to $\mathrm{p}$ with respect to $\mathrm{F}_{\mathrm{X}}$ is defined by

$$
\vec{H}_{\mathcal{B} / \mathrm{p} / \mathrm{X}} \triangleq \sum_{i=1}^{l} \vec{H}_{\mathcal{B}_{i} / \mathrm{p} / \mathrm{X}},
$$

where the angular momentum $\vec{H}_{\mathcal{B}_{i} / \mathrm{p} / \mathrm{X}}$ of $\mathcal{B}_{i}$ relative to $z$ with respect to $\mathrm{F}_{\mathrm{X}}$ is defined by

$$
\vec{H}_{\mathcal{B}_{i} / \mathrm{p} / \mathrm{X}} \triangleq \int_{\mathcal{B}_{i}} \vec{r}_{\mathrm{d} m / \mathrm{p}} \times \vec{v}_{\mathrm{d} m / \mathrm{p} / \mathrm{X}} \mathrm{d} m .
$$

Lemma 1. Let $\mathcal{B}$ be a rigid body, let $\mathrm{F}_{\mathrm{X}}$ and $\mathrm{F}_{\mathrm{Y}}$ be frames, and let $\mathrm{p}$ be a point. Then,

$$
\vec{H}_{\mathcal{B} / \mathrm{p} / \mathrm{X}}=\vec{I}_{\mathcal{B} / \mathrm{p}} \vec{\omega}_{\mathrm{Y} / \mathrm{X}}+\vec{H}_{\mathcal{B} / \mathrm{p} / \mathrm{Y}}
$$

where the physical inertia matrix $\vec{I}_{\mathcal{B} / \mathrm{p}}$ is defined by

$$
\vec{I}_{\mathcal{B} / \mathrm{p}} \triangleq \int_{\mathcal{B}}\left|\vec{r}_{\mathrm{d} m / \mathrm{p}}\right|^{2} \vec{U}-\vec{r}_{\mathrm{d} m / \mathrm{p}} \vec{r}_{\mathrm{d} m / \mathrm{p}}^{\prime} \mathrm{d} m
$$

where $\vec{U}$ is the second-order identity tensor.

Lemma 2. Let $\mathcal{B}$ be a rigid body, let $\mathrm{F}_{\mathrm{X}}$ and $\mathrm{F}_{\mathrm{Y}}$ be frames, let $\mathrm{F}_{\mathrm{Y}}$ be a body-fixed frame, and let $\mathrm{p}$ be a point that is fixed in $\mathcal{B}$. Then,

$$
\vec{H}_{\mathcal{B} / \mathrm{p} / \mathrm{Y}}=0
$$

and

$$
\vec{H}_{\mathcal{B} / \mathrm{p} / \mathrm{X}}=\vec{I}_{\mathcal{B} / \mathrm{p}} \vec{\omega}_{\mathrm{Y} / \mathrm{X}}
$$

Lemma 3. Let $F_{X}$ be a frame, let $\mathrm{p}$ be a point, let $\mathcal{B}$ be a rigid body with mass $m_{\mathcal{B}}$, and let $\mathrm{c}$ be the center of mass of $\mathcal{B}$. Then,

$$
\vec{H}_{\mathcal{B} / \mathrm{p} / \mathrm{X}}=\vec{H}_{\mathcal{B} / \mathrm{c} / \mathrm{X}}+\vec{r}_{\mathrm{c} / \mathrm{p}} \times m_{\mathcal{B}} \vec{v}_{\mathrm{c} / \mathrm{p} / \mathrm{X}} .
$$

We consider a spacecraft consisting of three single-axis CMGs with spherical gyro wheels attached to a rigid bus. Each CMG is mounted so that its gimbal is free to rotate about an axis passing through the center of mass of the gyro wheel. For simplicity, the gimbals are assumed to be massless. However, this paper does not assume that each gimbal's axis of rotation passes through the center of mass of the bus, nor does it assume that the CMGs are balanced with respect to the bus in order to preserve the location of its center of mass. Thus the center of mass of the spacecraft and the center of mass of the bus may be distinct points.

Let the spacecraft be denoted by sc, and let c denote its center of mass. Although the spacecraft is not a rigid body, the spherical symmetry of the gyro wheels implies that $\mathrm{c}$ is fixed in both the bus and the spacecraft. Let $\mathrm{c}_{i}$ denote the center of mass of the $i$ th gyro wheel. We assume a bus-fixed frame $\mathrm{F}_{\mathrm{B}}$, three gimbal-fixed frames $\mathrm{F}_{\mathrm{G}_{i}}$ whose $y$-axes are aligned with the rotation axes of their respective gimbals, three 
gyro wheel-fixed frames $\mathrm{F}_{\mathrm{W}_{i}}$ whose $x$-axes are aligned with the rotation axes of their respective gyro wheels, and an Earth-centered inertial frame $\mathrm{F}_{\mathrm{E}}$. The angular momentum of the spacecraft relative to its center of mass with respect to the inertial frame is given by

$$
\vec{H}_{\mathrm{sc} / \mathrm{c} / \mathrm{E}}=\vec{H}_{\mathrm{b} / \mathrm{c} / \mathrm{E}}+\sum_{i=1}^{3} \vec{H}_{\mathrm{w}_{i} / \mathrm{c} / \mathrm{E}}, \quad(\text { Def. } 1)
$$

where the angular momentum $\vec{H}_{\mathrm{b} / \mathrm{c} / \mathrm{E}}$ of the bus relative to $c$ with respect to $\mathrm{F}_{\mathrm{E}}$ is given by

$$
\vec{H}_{\mathrm{b} / \mathrm{c} / \mathrm{E}}=\vec{I}_{\mathrm{b} / \mathrm{c}} \vec{\omega}_{\mathrm{B} / \mathrm{E}}, \quad(\text { Lemma } 2)
$$

where $\vec{I}_{\mathrm{b} / \mathrm{c}}$ is the positive-definite inertia tensor of the bus relative to the center of mass of the spacecraft, and $\vec{\omega}_{\mathrm{B} / \mathrm{E}}$ is the angular velocity of $\mathrm{F}_{\mathrm{B}}$ with respect to $\mathrm{F}_{\mathrm{E}}$. The angular momentum $\vec{H}_{\mathrm{w}_{i} / \mathrm{c} / \mathrm{E}}$ of gyro wheel $i$ relative to the center of mass of the spacecraft with respect to the inertial frame is given by

$$
\begin{aligned}
\vec{H}_{\mathrm{w}_{i} / \mathrm{c} / \mathrm{E}} & =\vec{I}_{\mathrm{w}_{i} / \mathrm{c}} \vec{\omega}_{\mathrm{B} / \mathrm{E}}+\vec{H}_{\mathrm{w}_{i} / \mathrm{c} / \mathrm{B}} \\
& =\vec{I}_{\mathrm{w}_{i} / \mathrm{c}} \vec{\omega}_{\mathrm{B} / \mathrm{E}}+\vec{H}_{\mathrm{w}_{i} / \mathrm{c}_{i} / \mathrm{B}}+\vec{r}_{\mathrm{c}_{i} / \mathrm{c}} \times m_{\mathrm{w}_{i}} \vec{v}_{\mathrm{c}_{i} / \mathrm{c} / \mathrm{B}} \\
& =\vec{I}_{\mathrm{w}_{i} / \mathrm{c}} \vec{\omega}_{\mathrm{B} / \mathrm{E}}+\vec{I}_{\mathrm{w}_{i} / \mathrm{c}_{i}} \vec{\omega}_{\mathrm{W}_{i} / \mathrm{B}},
\end{aligned}
$$

where $\vec{I}_{\mathrm{w}_{i} / \mathrm{c}}$ is the positive-definite inertia tensor of gyro wheel $i$ relative to the spacecraft's center of mass, $\vec{I}_{\mathrm{w}_{i} / \mathrm{c}_{i}}$ is the positive-definite inertia tensor of gyro wheel $i$ relative to its own center of mass $\mathrm{c}_{i}$, and $\vec{\omega}_{\mathrm{W}_{i} / \mathrm{B}}$ is the angular velocity of gyro wheel $i$ relative to the bus, and is given by

$$
\vec{\omega}_{\mathrm{W}_{i} / \mathrm{B}}=\vec{\omega}_{\mathrm{W}_{i} / \mathrm{G}_{i}}+\vec{\omega}_{\mathrm{G}_{i} / \mathrm{B}}
$$

where $\vec{\omega}_{\mathrm{W}_{i} / \mathrm{G}_{i}}$ is the angular velocity of gyro wheel $i$ relative to the gimbal $i$, and $\vec{\omega}_{\mathrm{G}_{i} / \mathrm{B}}$ is the angular velocity of gimbal $i$ relative to the bus. Thus (8) is given by

$$
\vec{H}_{\mathrm{sc} / \mathrm{c} / \mathrm{E}}=\left(\vec{I}_{\mathrm{b} / \mathrm{c}}+\sum_{i=1}^{3} \vec{I}_{\mathrm{w}_{i} / \mathrm{c}}\right) \vec{\omega}_{\mathrm{B} / \mathrm{E}}+\sum_{i=1}^{3} \vec{I}_{\mathrm{w}_{i} / \mathrm{c}_{i}} \vec{\omega}_{\mathrm{W}_{i} / \mathrm{B}}
$$

Note that $\vec{\omega}_{\mathrm{W}_{i} / \mathrm{B}}$ has only two degrees of freedom relative to the bus, which can be seen when resolving $\vec{\omega}_{\mathrm{W}_{i} / \mathrm{G}_{i}}$ and $\vec{\omega}_{\mathrm{G}_{i} / \mathrm{B}}$ in $\mathrm{F}_{\mathrm{W}_{i}}$ and $\mathrm{F}_{\mathrm{G}_{i}}$, respectively, that is,

$$
\left.\vec{\omega}_{\mathrm{W}_{i} / \mathrm{G}_{i}}\right|_{\mathrm{W}_{i}}=\left[\begin{array}{c}
q_{i} \\
0 \\
0
\end{array}\right],\left.\quad \vec{\omega}_{\mathrm{G}_{i} / \mathrm{B}}\right|_{\mathrm{G}_{i}}=\left[\begin{array}{c}
0 \\
p_{i} \\
0
\end{array}\right] .
$$

Since $\mathrm{F}_{\mathrm{W}_{i}}$ is aligned with the principal axes of gyro wheel $i$, we have

$$
\left.\vec{I}_{\mathrm{w}_{i} / \mathrm{c}_{i}}\right|_{\mathrm{W}_{i}}=\left[\begin{array}{ccc}
\beta_{i} & 0 & 0 \\
0 & \beta_{i} & 0 \\
0 & 0 & \beta_{i}
\end{array}\right]
$$

The repeated entries are due to the spherical nature of the gyro wheels. $\mathrm{F}_{\mathrm{W}_{i}}$ rotates relative to $\mathrm{F}_{\mathrm{G}_{i}}$ about its $x$-axis. Consequently,

$$
\left.\vec{I}_{\mathrm{w}_{i} / \mathrm{c}_{i}}\right|_{\mathrm{G}_{i}}=\left.\vec{I}_{\mathrm{w}_{i} / \mathrm{c}_{i}}\right|_{\mathrm{W}_{i}}
$$


Therefore,

$$
\overbrace{\vec{I}_{\mathrm{w}_{i} / \mathrm{c}_{i}}}^{\mathrm{G}_{i} \bullet}=0 .
$$

Note that $\vec{\omega}_{\mathrm{W}_{i} / \mathrm{G}_{i}}$ is an eigenvector of $\vec{I}_{\mathrm{w}_{i} / \mathrm{c}_{i}}$ with eigenvalue $\beta_{i}$, and $\vec{\omega}_{\mathrm{G}_{i} / \mathrm{B}}$ is an eigenvector of $\vec{I}_{\mathrm{w}_{i} / \mathrm{c}_{i}}$ with eigenvalue $\beta_{i}$. That is, $\vec{I}_{\mathrm{w}_{i} / \mathrm{c}_{i}} \vec{\omega}_{\mathrm{W}_{i} / \mathrm{G}_{i}}=\beta_{i} \vec{\omega}_{\mathrm{W}_{i} / \mathrm{G}_{i}}$ and $\vec{I}_{\mathrm{w}_{i} / \mathrm{c}_{i}} \vec{\omega}_{\mathrm{G}_{i} / \mathrm{B}}=\beta_{i} \vec{\omega}_{\mathrm{G}_{i} / \mathrm{B}}$.

\section{Spacecraft Equations of Motion}

We now derive the equations of motion for a spacecraft actuated with CMGs as described above. It follows from Newton's second law for rotation, that

$$
\begin{aligned}
\vec{M}_{\mathrm{sc} / \mathrm{c}}= & \overbrace{\left(\vec{H}_{\mathrm{sc} / \mathrm{c} / \mathrm{E}}^{\mathrm{E}}{ }^{\mathrm{b} / \mathrm{c}}+\sum_{i=1}^{3} \vec{I}_{\mathrm{w}_{i} / \mathrm{c}}\right) \vec{\omega}_{\mathrm{B} / \mathrm{E}}}^{\mathrm{E} \bullet}+\overbrace{\sum_{i=1}^{3} \vec{I}_{\mathrm{w}_{i} / \mathrm{c}_{i}} \vec{\omega}_{\mathrm{W}_{i} / \mathrm{B}}}^{\mathrm{E} \bullet}+\overbrace{\left(\vec{I}_{\mathrm{b} / \mathrm{c}}+\sum_{i=1}^{3} \vec{I}_{\mathrm{w}_{i} / \mathrm{c}}\right) \vec{\omega}_{\mathrm{B} / \mathrm{E}}}^{\mathrm{B} \bullet} \vec{\omega}_{\mathrm{B} / \mathrm{E}} \times\left(\vec{I}_{\mathrm{b} / \mathrm{c}}+\sum_{i=1}^{3} \vec{I}_{\mathrm{w}_{i} / \mathrm{c}}\right) \vec{\omega}_{\mathrm{B} / \mathrm{E}} \\
= & \overbrace{\left(\sum_{i=1}^{3} \vec{I}_{\mathrm{w}_{i} / \mathrm{c}_{i}}+\vec{\omega}_{\mathrm{W}_{i} / \mathrm{B}}+\sum_{i=1}^{3} \vec{\omega}_{\mathrm{B} / \mathrm{E}} \times \sum_{i=1}^{3}\right) \vec{I}_{\mathrm{w}_{i} / \mathrm{c} / \mathrm{c}} \vec{\omega}_{\mathrm{W}_{i} / \mathrm{B}}^{\mathrm{B} \bullet}}^{\mathrm{B}}+\sum_{\mathrm{B} / \mathrm{E}}^{3} \beta_{i=1} \vec{\omega}_{\mathrm{W}_{i} / \mathrm{G}_{i}}+\sum_{i=1}^{3} \beta_{i} \vec{\omega}_{\mathrm{G}_{i} / \mathrm{B}}^{\mathrm{B} \bullet} \\
& +\vec{\omega}_{\mathrm{B} / \mathrm{E}} \times\left(\left(\vec{I}_{\mathrm{b} / \mathrm{c}}+\sum_{i=1}^{3} \vec{I}_{\mathrm{w}_{i} / \mathrm{c}}\right) \vec{\omega}_{\mathrm{B} / \mathrm{E}}+\sum_{i=1}^{3} \beta_{i}\left(\vec{\omega}_{\mathrm{W}_{i} / \mathrm{G}_{i}}+\vec{\omega}_{\mathrm{G}_{i} / \mathrm{B}}\right)\right) .
\end{aligned}
$$

Rearranging (17) yields

$$
\begin{aligned}
\left(\vec{I}_{\mathrm{b} / \mathrm{c}}+\sum_{i=1}^{3} \vec{I}_{\mathrm{w}_{i} / \mathrm{c}}\right) \vec{\omega}_{\mathrm{B} / \mathrm{E}}^{\mathrm{B} \bullet}=( & \left.\left(\vec{I}_{\mathrm{b} / \mathrm{c}}+\sum_{i=1}^{3} \vec{I}_{\mathrm{w}_{i} / \mathrm{c}}\right) \vec{\omega}_{\mathrm{B} / \mathrm{E}}+\sum_{i=1}^{3} \beta_{i}\left(\vec{\omega}_{\mathrm{W}_{i} / \mathrm{G}_{i}}+\vec{\omega}_{\mathrm{G}_{i} / \mathrm{B}}\right)\right) \times \vec{\omega}_{\mathrm{B} / \mathrm{E}} \\
& -\sum_{i=1}^{3} \beta_{i} \vec{\omega}_{\mathrm{W}_{i} / \mathrm{G}_{i}}^{\mathrm{B} \bullet}-\sum_{i=1}^{3} \beta_{i} \vec{\omega}_{\mathrm{G}_{i} / \mathrm{B}}^{\mathrm{B}}+\vec{M}_{\mathrm{sc} / \mathrm{c}} .
\end{aligned}
$$


To resolve (18) in $\mathrm{F}_{\mathrm{B}}$, the following notation is used:

$$
\begin{aligned}
& \left.J_{\mathrm{b}} \triangleq \vec{I}_{\mathrm{b} / \mathrm{c}}\right|_{\mathrm{B}}, \\
& \left.J_{\mathrm{w}_{i} / \mathrm{c}} \triangleq \vec{I}_{\mathrm{w}_{i} / \mathrm{c}}\right|_{\mathrm{B}}, \\
& \left.J_{\mathrm{w}_{i} / \mathrm{c}_{i}} \triangleq \vec{I}_{\mathrm{w}_{i} / \mathrm{c}_{i}}\right|_{\mathrm{B}}, \\
& J_{\mathrm{sc}} \triangleq J_{\mathrm{b}}+\sum_{i=1}^{3} J_{\mathrm{w}_{i} / \mathrm{c}}, \\
& \left.\omega \triangleq \vec{\omega}_{\mathrm{B} / \mathrm{E}}\right|_{\mathrm{B}}, \\
& \left.\dot{\omega} \triangleq \stackrel{\mathrm{B}}{\vec{\omega}}_{\mathrm{B} / \mathrm{E}}\right|_{\mathrm{B}}, \\
& \left.\nu_{i} \triangleq \vec{\omega}_{\mathrm{G}_{i} / \mathrm{B}}\right|_{\mathrm{B}}, \\
& \left.\dot{\nu}_{i} \triangleq \stackrel{\mathrm{B} \bullet}{\vec{\omega}}_{\mathrm{G}_{i} / \mathrm{B}}\right|_{\mathrm{B}}, \\
& \left.\omega_{\mathrm{W}_{i}} \triangleq \vec{\omega}_{\mathrm{W}_{i} / \mathrm{G}_{i}}\right|_{\mathrm{B}}, \\
& \left.\dot{\omega}_{\mathrm{W}_{i}} \triangleq \stackrel{\mathrm{B} \bullet}{\vec{\omega}}_{\mathrm{W}_{i} / \mathrm{G}_{i}}\right|_{\mathrm{B}}, \\
& \left.\tau_{\mathrm{dist}} \triangleq \vec{M}_{\mathrm{sc} / \mathrm{c}}\right|_{\mathrm{B}} .
\end{aligned}
$$

We let the vector $\tau_{\text {dist }}$ represent disturbance torques, that is, all internal and external torques applied to the spacecraft aside from control torques. Disturbance torques may be due to onboard components, gravity gradients, solar pressure, atmospheric drag, or the ambient magnetic field.

Resolving (18) in $\mathrm{F}_{\mathrm{B}}$ yields the equation of motion for a spacecraft with CMGs, which has the form

$$
J_{\mathrm{sc}} \dot{\omega}=\left(J_{\mathrm{sc}} \omega+\sum_{i=1}^{3} \beta_{i}\left(\nu_{i}+\omega_{\mathrm{W}_{i}}\right)\right) \times \omega-\sum_{i=1}^{3} \beta_{i} \dot{\nu}_{i}-\sum_{i=1}^{3} \beta_{i} \dot{\omega}_{\mathrm{W}_{i}}+\tau_{\text {dist }} .
$$

\section{A. Special Case: gyro wheels rotate with constant angular velocity}

We now specialize the equation of motion (19) by assuming that the gyro wheels rotate with a constant angular velocity. We thus have,

$$
\stackrel{\mathrm{W}_{i} \bullet}{\vec{\omega}} \mathrm{W}_{i} / \mathrm{G}_{i}=0 .
$$

Then,

$$
\begin{aligned}
\stackrel{\mathrm{B} \bullet}{\omega}_{\mathrm{W}_{i} / \mathrm{G}_{i}} & =\stackrel{\mathrm{G}_{i} \bullet}{\vec{\omega}} \mathrm{W}_{i} / \mathrm{G}_{i} \\
& +\vec{\omega}_{\mathrm{G}_{i} / \mathrm{B}} \times \vec{\omega}_{\mathrm{W}_{i} / \mathrm{G}_{i}} \\
& =\vec{\omega}_{\mathrm{W}_{i} / \mathrm{G}_{i}}+\vec{\omega}_{\mathrm{G}_{i} / \mathrm{B}} \times \vec{\omega}_{\mathrm{W}_{i} / \mathrm{G}_{i}} \\
& =\vec{\omega}_{\mathrm{G}_{i} / \mathrm{B}} \times \vec{\omega}_{\mathrm{W}_{i} / \mathrm{G}_{i}} .
\end{aligned}
$$


Substituting (21) into (18) and resolving in $\mathrm{F}_{\mathrm{B}}$ yields

$$
J_{\mathrm{sc}} \dot{\omega}=\left(J_{\mathrm{sc}} \omega+\sum_{i=1}^{3} \beta_{i}\left(\nu_{i}+\omega_{\mathrm{W}_{i}}\right)\right) \times \omega-\sum_{i=1}^{3} \beta_{i} \dot{\nu}_{i}-\sum_{i=1}^{3} \beta_{i}\left(\nu_{i} \times \omega_{\mathrm{W}_{i}}\right)+\tau_{\text {dist }} .
$$

\section{B. Special case: orthogonal gimbals aligned with $F_{B}$}

We further specialize the equations of motion by assuming that the gimbals are mounted orthogonally to each other, but their rotation axes need not intersect. For further simplification, we align $F_{B}$ with the rotational axes of the three orthogonal gimbals. However, we do not assume that $\mathrm{F}_{\mathrm{B}}$ is aligned with the principal axes of the bus. In this configuration

$$
\mathcal{O}_{\mathrm{B} / \mathrm{G}_{1}} e_{2}=\left[\begin{array}{l}
1 \\
0 \\
0
\end{array}\right]=e_{1}, \quad \mathcal{O}_{\mathrm{B} / \mathrm{G}_{2}} e_{2}=\left[\begin{array}{l}
0 \\
1 \\
0
\end{array}\right]=e_{2}, \quad \mathcal{O}_{\mathrm{B} / \mathrm{G}_{3}} e_{2}=\left[\begin{array}{l}
0 \\
0 \\
1
\end{array}\right]=e_{3},
$$

where $\mathcal{O}_{\mathrm{B} / \mathrm{G}_{i}} \in \mathbb{R}^{3 \times 3}$ is the proper orthogonal matrix (that is, the orientation matrix) that transforms the components of a vector resolved in $\mathrm{F}_{\mathrm{G}_{i}}$ into the components of the same vector resolved in $\mathrm{F}_{\mathrm{B}}$.

Additionally, as we will be directly commanding the gimbal angular rates, we rearrange (18) in order to group all terms involving $\vec{\omega}_{\mathrm{G}_{i} / \mathrm{B}}$, which we now write as $u_{i}$. We thus have

$$
\begin{aligned}
& \left(\vec{I}_{\mathrm{b} / \mathrm{c}}+\sum_{i=1}^{3} \vec{I}_{\mathrm{w}_{i} / \mathrm{c}}\right) \vec{\omega}_{\mathrm{B} / \mathrm{E}}^{\mathrm{B} \bullet}=\left(\left(\vec{I}_{\mathrm{b} / \mathrm{c}}+\sum_{i=1}^{3} \vec{I}_{\mathrm{w}_{i} / \mathrm{c}}\right) \vec{\omega}_{\mathrm{B} / \mathrm{E}}+\sum_{i=1}^{3} \beta_{i}\left(\vec{\omega}_{\mathrm{W}_{i} / \mathrm{G}_{i}}+\vec{\omega}_{\mathrm{G}_{i} / \mathrm{B}}\right)\right) \times \vec{\omega}_{\mathrm{B} / \mathrm{E}} \\
& -\sum_{i=1}^{3} \beta_{i}\left(\vec{\omega}_{\mathrm{G}_{i} / \mathrm{B}} \times \vec{\omega}_{\mathrm{W}_{i} / \mathrm{G}_{i}}\right)-\sum_{i=1}^{3} \beta_{i} \vec{\omega}_{\mathrm{G}_{i} / \mathrm{B}}^{\mathrm{B} \bullet}+\vec{M}_{\mathrm{sc} / \mathrm{c}} \\
& =\left(\left(\vec{I}_{\mathrm{b} / \mathrm{c}}+\sum_{i=1}^{3} \vec{I}_{\mathrm{w}_{i} / \mathrm{c}}\right) \vec{\omega}_{\mathrm{B} / \mathrm{E}}+\sum_{i=1}^{3} \beta_{i} \vec{\omega}_{\mathrm{W}_{i} / \mathrm{G}_{i}}\right) \times \vec{\omega}_{\mathrm{B} / \mathrm{E}}-\sum_{i=1}^{3} \beta_{i} \vec{\omega}_{\mathrm{B} / \mathrm{E}}^{\times} \vec{\omega}_{\mathrm{G}_{i} / \mathrm{B}} \\
& +\sum_{i=1}^{3} \beta_{i} \vec{\omega}_{\mathrm{W}_{i} / \mathrm{G}_{i}}^{\times} \vec{\omega}_{\mathrm{G}_{i} / \mathrm{B}}-\sum_{i=1}^{3} \beta_{i} \vec{\omega}_{\mathrm{G}_{i} / \mathrm{B}}^{\mathrm{B} \bullet}+\vec{M}_{\mathrm{sc} / \mathrm{c}} \\
& =\left(\left(\vec{I}_{\mathrm{b} / \mathrm{c}}+\sum_{i=1}^{3} \vec{I}_{\mathrm{w}_{i} / \mathrm{c}}\right) \vec{\omega}_{\mathrm{B} / \mathrm{E}}+\sum_{i=1}^{3} \beta_{i} \vec{\omega}_{\mathrm{W}_{i} / \mathrm{G}_{i}}\right) \times \vec{\omega}_{\mathrm{B} / \mathrm{E}} \\
& +\sum_{i=1}^{3} \beta_{i}\left(\vec{\omega}_{\mathrm{W}_{i} / \mathrm{G}_{i}}^{\times}-\vec{\omega}_{\mathrm{B} / \mathrm{E}}^{\times}\right) \mathcal{O}_{\mathrm{B} / \mathrm{G}_{i}} e_{2} u_{i}-\sum_{i=1}^{3} \beta_{i} \vec{\omega}_{\mathrm{G}_{i} / \mathrm{B}}^{\mathrm{B} \bullet}+\vec{M}_{\mathrm{sc} / \mathrm{c}} .
\end{aligned}
$$

Expanding the control term in (24) and plugging in (23) yields,

$$
\begin{aligned}
& \sum_{i=1}^{3} \beta_{i}\left(\vec{\omega}_{\mathrm{W}_{i} / \mathrm{G}_{i}}^{\times}-\vec{\omega}_{\mathrm{B} / \mathrm{E}}^{\times}\right) \mathcal{O}_{\mathrm{B} / \mathrm{G}_{i}} e_{2} u_{i} \\
& \quad=\beta_{1}\left(\vec{\omega}_{\mathrm{W}_{1} / \mathrm{G}_{1}}^{\times}-\vec{\omega}_{\mathrm{B} / \mathrm{E}}^{\times}\right) e_{1} u_{1}+\beta_{2}\left(\vec{\omega}_{\mathrm{W}_{2} / \mathrm{G}_{2}}^{\times}-\vec{\omega}_{\mathrm{B} / \mathrm{E}}^{\times}\right) e_{2} u_{2}+\beta_{3}\left(\vec{\omega}_{\mathrm{W}_{3} / \mathrm{G}_{3}}^{\times}-\vec{\omega}_{\mathrm{B} / \mathrm{E}}^{\times}\right) e_{3} u_{3} \\
& \quad=Y u,
\end{aligned}
$$


where



Finally, resolving the equation of motion (24) in $\mathrm{F}_{\mathrm{B}}$ yields

$$
J_{\mathrm{sc}} \dot{\omega}=\left(J_{\mathrm{sc}} \omega+\sum_{i=1}^{3} \beta_{i} \omega_{\mathrm{W}_{i}}\right) \times \omega+Y u-J_{\beta} \dot{u}+\tau_{\mathrm{dist}},
$$

where,

$$
J_{\beta}=\left[\begin{array}{ccc}
\beta_{1} & 0 & 0 \\
0 & \beta_{2} & 0 \\
0 & 0 & \beta_{3}
\end{array}\right] \text {. }
$$

\section{Spacecraft Model, Assumptions, and Objectives for Control Design}

The kinematics of the spacecraft model are given by Poisson's equation

$$
\dot{R}=R \omega^{\times},
$$

which complements $(26)$. In $(28), \omega^{\times}$denotes the skew-symmetric matrix of $\omega$, and $R \triangleq \mathcal{O}_{\mathrm{E} / \mathrm{B}} \in \mathbb{R}^{3 \times 3}$.

Compared to the case of reaction wheels treated in ref., ${ }^{2}$ control moment gyroscope actuation complicates the dynamic equations due to the term $J_{\beta} \dot{u}$ in (26), as well as the state-dependant, time-varying, and potentially singular input matrix, $Y$. The kinematic relation (28) remains unchanged. The torque inputs applied to each gyro wheel are constrained by current limitations on the electric motors and amplifiers as well as angular-velocity constraints on the gimbals. However, these constraints are not addressed explicitly.

Both rate (inertial) and attitude (noninertial) measurements are assumed to be available. Gyro measurements $y_{\text {rate }} \in \mathbb{R}^{3}$ are assumed to provide measurements of the angular velocity resolved in the spacecraft frame, that is,

$$
y_{\text {rate }}=\omega+v_{\text {rate }},
$$

where $v_{\text {rate }} \in \mathbb{R}^{3}$ represents the presence of noise in the gyro measurements. Attitude is measured indirectly using sensors such as magnetometers or star trackers. The attitude is determined to be

$$
y_{\text {attitude }}=R \text {. }
$$

When attitude measurements are given in terms of an alternative attitude representation, such as quaternions, Rodrigues's formula can be used to determine the corresponding rotation matrix. Attitude estimation on $\mathrm{SO}(3)$ is considered in ref. ${ }^{20}$

The objective of the attitude control problem is to determine control inputs such that the spacecraft attitude given by $R$ follows a commanded attitude trajectory given by a possibly time-varying $\mathrm{C}^{1}$ rotation matrix $R_{\mathrm{d}}(t)$. For $t \geq 0, R_{\mathrm{d}}(t)$ is given by

$$
\begin{aligned}
\dot{R}_{\mathrm{d}}(t) & =R_{\mathrm{d}}(t) \omega_{\mathrm{d}}(t)^{\times}, \\
R_{\mathrm{d}}(0) & =R_{\mathrm{d} 0},
\end{aligned}
$$


where $\omega_{\mathrm{d}}$ is the desired, possibly time-varying angular velocity. The error between $R(t)$ and $R_{\mathrm{d}}(t)$ is given in terms of the attitude-error rotation matrix

$$
\tilde{R} \triangleq R_{\mathrm{d}}^{\mathrm{T}} R
$$

which satisfies the differential equation

$$
\dot{\tilde{R}}=\tilde{R} \tilde{\omega}^{\times},
$$

where the angular velocity error $\tilde{\omega}$ is defined by

$$
\tilde{\omega} \triangleq \omega-\tilde{R}^{\mathrm{T}} \omega_{\mathrm{d}}
$$

We rewrite (26) in terms of the angular-velocity error as

$$
\begin{aligned}
J_{\mathrm{sc}} \dot{\tilde{\omega}}= & \left(J_{\mathrm{sc}}\left(\tilde{\omega}+\tilde{R}^{\mathrm{T}} \omega_{\mathrm{d}}\right)+\sum_{i=1}^{3} \beta_{i} \omega_{\mathrm{W}_{i}}\right) \times\left(\tilde{\omega}+\tilde{R}^{\mathrm{T}} \omega_{\mathrm{d}}\right)+J_{\mathrm{sc}}\left(\tilde{\omega} \times \tilde{R}^{\mathrm{T}} \omega_{\mathrm{d}}-\tilde{R}^{\mathrm{T}} \dot{\omega}_{\mathrm{d}}\right) \\
& +Y u-J_{\beta} \dot{u}+\tau_{\text {dist }} .
\end{aligned}
$$

A scalar measure of attitude error is given by the rotation angle $\theta(t)$ about an eigenaxis needed to rotate the spacecraft from its attitude $R(t)$ to the desired attitude $R_{\mathrm{d}}(t)$. This angle is given by ${ }^{19}$

$$
\theta(t)=\cos ^{-1}\left(\frac{1}{2}[\operatorname{tr} \tilde{R}(t)-1]\right),
$$

and referred to as the eigenaxis attitude error.

\section{Controller Design}

Let $I$ denote the identity matrix, whose dimensions are determined by context, and let $M_{i j}$ denote the $i, j$ entry of the matrix $M$. The following result is given in ref. ${ }^{1}$

Lemma 4. Let $A \in \mathbb{R}^{3 \times 3}$ be a diagonal positive-definite matrix. Then the following statements hold for a proper orthogonal matrix $R$ :

i) For all $i, j=1,2,3, R_{i j} \in[-1,1]$.

ii) $\operatorname{tr}(A-A R) \geq 0$.

iii) $\operatorname{tr}(A-A R)=0$ if and only if $R=I$.

For convenience we note that, if $R$ is a rotation matrix and $x, y \in \mathbb{R}^{3}$, then

$$
(R x)^{\times}=R x^{\times} R^{\mathrm{T}},
$$

and, therefore,

$$
R(x \times y)=(R x) \times R y .
$$

Next we introduce the notation

$$
J_{\mathrm{sc}} \omega=L(\omega) \gamma
$$

where $\gamma \in \mathbb{R}^{6}$ is defined by

$$
\gamma \triangleq\left[\begin{array}{llllll}
J_{11} & J_{22} & J_{33} & J_{23} & J_{13} & J_{12}
\end{array}\right]^{\mathrm{T}}
$$

and

$$
L(\omega) \triangleq\left[\begin{array}{cccccc}
\omega_{1} & 0 & 0 & 0 & \omega_{3} & \omega_{2} \\
0 & \omega_{2} & 0 & \omega_{3} & 0 & \omega_{1} \\
0 & 0 & \omega_{3} & \omega_{2} & \omega_{1} & 0
\end{array}\right]
$$


Next, let $\hat{J}_{\mathrm{sc}} \in \mathbb{R}^{3 \times 3}$ denote an estimate of $J_{\mathrm{sc}}$, and define the inertia-estimation error

$$
\tilde{J}_{\mathrm{sc}} \triangleq J_{\mathrm{sc}}-\hat{J}_{\mathrm{sc}} .
$$

Letting $\hat{\gamma}, \tilde{\gamma} \in \mathbb{R}^{6}$ represent $\hat{J}_{\mathrm{sc}}, \tilde{J}_{\mathrm{sc}}$, respectively, it follows that

$$
\tilde{\gamma}=\gamma-\hat{\gamma}
$$

Likewise, let $\hat{\tau}_{\text {dist }} \in \mathbb{R}^{3}$ denote an estimate of $\tau_{\text {dist }}$, and define the disturbance-estimation error

$$
\tilde{\tau}_{\text {dist }} \triangleq \tau_{\text {dist }}-\hat{\tau}_{\text {dist }} \text {. }
$$

The assumptions upon which the following development is based are now stated.

Assumption 1. $J_{\mathrm{sc}}$ is constant but unknown.

Assumption 2. $J_{\beta}$ is constant, nonsingular, and known. That is, we have three orthogonal CMGs with symmetric gyro wheels, and we know the moments of inertia about their spin axes.

Assumption 3. $\dot{u}$, the gimbal accelerations, are neglegible and can be ignored. We will ignore the effect of $\dot{u}$ in our derivation of the control law. This treatment of $\dot{u}$ is consistent with prior literature. ${ }^{21-23} \mathrm{We}$ will however, consider it in the actual plant dynamics for simulations and show through numerical examples that this is a reasonable assumption. Removing $\dot{u}$ from (26) yields

$$
J_{\mathrm{sc}} \dot{\omega}=\left(J_{\mathrm{sc}} \omega+\sum_{i=1}^{3} \beta_{i} \omega_{\mathrm{W}_{i}}\right) \times \omega+Y u+\tau_{\text {dist }} .
$$

Two controllers are presented in ref. ${ }^{1}$ They are now modified, as necessary, for control moment gyroscrope actuation.

\section{A. Control Law for Slew Maneuvers}

When no disturbances are present, the inertia-free control law given by (38) of ref. ${ }^{1}$ achieves almost global stabilization of a constant desired attitude $R_{\mathrm{d}}$, that is, a slew maneuver that brings the spacecraft to rest. The initial conditions of the slew maneuver may be arbitrary, that is, the spacecraft may have nonzero initial velocity.

Given $a=\left[a_{1} a_{2} a_{3}\right]^{\mathrm{T}} \in \mathbb{R}^{3}$ such that $a_{1}, a_{2}$ and $a_{3}$ are positive and distinct, define the vector measure of attitude error

$$
S \triangleq \sum_{i=1}^{3} a_{i}\left(\tilde{R}^{\mathrm{T}} e_{i}\right) \times e_{i}
$$

where, for $i=1,2,3, e_{i}$ denotes the $i$ th column of the $3 \times 3$ identity matrix.

Theorem 1. Let $K_{\mathrm{p}}$ be a positive number and let $A=\operatorname{diag}(a)$ be a diagonal positive-definite matrix. Then the function

$$
V(\omega, \tilde{R}) \triangleq \frac{1}{2} \omega^{T} J_{\mathrm{sc}} \omega+K_{\mathrm{p}} \operatorname{tr}(A-A \tilde{R}),
$$

is positive definite, that is, $V$ is nonnegative, and $V=0$ if and only if $\omega=0$ and $\tilde{R}=I$,.

Proof. It follows from statement 2 of Lemma 4 that $\operatorname{tr}(A-A \tilde{R})$ is nonnegative. Hence $V$ is nonnegative. Now suppose that $V=0$. Then, $\omega=0$, and it follows from statement 3 of Lemma 4 that $\tilde{R}=I$.

Note that (38), which we intend to use as a Lyapunov function, is not positive in all the states, namely, it makes no use of the relative angular velocity of the gyro wheels or the gimbals since gyro wheel-speed regulation and gimbal-speed regulation are not goals of the control objective. 
Theorem 2. Let $K_{\mathrm{p}}$ be a positive number, let $K_{\mathrm{v}} \in \mathbb{R}^{3 \times 3}$ be a positive-definite matrix, let $A=$ $\operatorname{diag}\left(a_{1}, a_{2}, a_{3}\right)$ be a diagonal positive-definite matrix, define $S$ as in (37), and define $V$ as in Theorem 1. Furthermore, consider the control law

$$
u=-Y^{-1}\left(K_{\mathrm{p}} S+K_{\mathrm{v}} \omega\right),
$$

and assume that $Y$ is nonsingular. Then,

$$
\dot{V}(\omega, \tilde{R})=-\omega^{T} K_{\mathrm{v}} \omega
$$

along the trajectories of (36), is negative semidefinite.

Proof. Noting that

$$
\begin{aligned}
\frac{\mathrm{d}}{\mathrm{d} t} \operatorname{tr}(A-A \tilde{R}) & =-\operatorname{tr} A \dot{\tilde{R}} \\
& =-\operatorname{tr} A\left(\tilde{R} \omega^{\times}-\omega_{\mathrm{d}}^{\times} \tilde{R}\right) \\
& =-\sum_{i=1}^{3} a_{i} e_{i}^{\mathrm{T}}\left(\tilde{R} \omega^{\times}-\omega_{\mathrm{d}}^{\times} \tilde{R}\right) e_{i} \\
& =-\sum_{i=1}^{3} a_{i} e_{i}^{\mathrm{T}} \tilde{R}\left(\omega^{\times}-\tilde{R}^{\mathrm{T}} \omega_{\mathrm{d}}^{\times} \tilde{R}\right) e_{i} \\
& =-\sum_{i=1}^{3} a_{i} e_{i}^{\mathrm{T}} \tilde{R}\left(\omega-\tilde{R}^{\mathrm{T}} \omega_{\mathrm{d}}\right)^{\times} e_{i} \\
& =\sum_{i=1}^{3} a_{i} e_{i}^{\mathrm{T}} \tilde{R} e_{i}^{\times} \tilde{\omega} \\
& =\left[-\sum_{i=1}^{3} a_{i} e_{i} \times \tilde{R}^{\mathrm{T}} e_{i}\right]^{\mathrm{T}} \tilde{\omega} \\
& =\left[\sum_{i=1}^{3} a_{i}\left(\tilde{R}^{\mathrm{T}} e_{i}\right) \times e_{i}\right]^{\mathrm{T}} \tilde{\omega} \\
& =\tilde{\omega}^{\mathrm{T}} S
\end{aligned}
$$

then

$$
\begin{aligned}
\dot{V}(\omega, \tilde{R}) & =\omega^{T} J_{\mathrm{sc}} \dot{\omega}+K_{\mathrm{p}} \omega^{T} S \\
& =\omega^{T}\left[\left(J_{\mathrm{sc}} \omega+\sum_{i=1}^{3} \beta_{i} \omega_{\mathrm{W}_{i}}\right) \times \omega+Y u\right]+K_{\mathrm{p}} \omega^{T} S \\
& =\omega^{T}\left(-K_{\mathrm{p}} S-K_{\mathrm{v}} \omega\right)+K_{\mathrm{p}} \omega^{T} S \\
& =-\omega^{T} K_{\mathrm{v}} \omega .
\end{aligned}
$$

Note that $-Y$ is substituted for the input matrix B used in the inertia-free control law (38) of ref. ${ }^{1}$ but otherwise the controller requires no modification for the case of control moment gyroscope actuation.

\section{B. Control Law for Attitude Tracking}

A control law that tracks a desired attitude trajectory in the presence of disturbances is given by (21) of ref. ${ }^{1}$ This controller is based on an additional assumption.

Assumption 4. Each component of $\tau_{\text {dist }}$ is a linear combination of constant and harmonic signals, whose frequencies are known but whose amplitudes and phases are unknown.

Assumption 4 implies that $\tau_{\text {dist }}$ can be modeled as the output of an autonomous system of the form

$$
\begin{aligned}
\dot{d} & =A_{d} d, \\
\tau_{\text {dist }} & =C_{d} d,
\end{aligned}
$$


where $A_{d} \in \mathbb{R}^{n_{d} \times n_{d}}$ and $C_{d} \in \mathbb{R}^{3 \times n_{d}}$ are known matrices and $A_{d}$ is a Lyapunov-stable matrix. In this model, $d(0)$ is unknown, which is equivalent to the assumption that the amplitude and phase of each harmonic component of the disturbance is unknown. The matrix $A_{d}$ is chosen to include eigenvalues of all frequency components that may be present in the disturbance signal, where the zero eigenvalue corresponds to a constant disturbance. In effect, the controller provides infinite gain at the disturbance frequency, which results in asymptotic rejection of harmonic disturbance components. In particular, an integral controller provides infinite gain at DC in order to reject constant disturbances. In the case of orbit-dependent disturbances, the frequencies can be estimated from the orbital parameters. Likewise, in the case of disturbances originating from on-board devices, the spectral content of the disturbances may be known. In other cases, it may be possible to estimate the spectrum of the disturbances through signal processing. Assumption 4 implies that $A_{d}$ can be chosen to be skew symmetric, which we do henceforth. Let $\hat{d} \in \mathbb{R}^{n_{d}}$ denote an estimate of $d$, and define the disturbance-state estimation error

$$
\tilde{d} \triangleq d-\hat{d}
$$

The attitude tracking controller in the presence of disturbances is modified for CMG actuators below.

Theorem 3. Let $K_{\mathrm{p}}$ be a positive number, let $K_{1} \in \mathbb{R}^{3 \times 3}$, let $Q \in \mathbb{R}^{6 \times 6}$ and $D \in \mathbb{R}^{n_{d} \times n_{d}}$ be positive definite matrices, let $A=\operatorname{diag}\left(a_{1}, a_{2}, a_{3}\right)$ be a diagonal positive-definite matrix, and define $S$ as before. Then the function

$$
V(\tilde{\omega}, \tilde{R}, \tilde{\gamma}, \tilde{d}) \triangleq \frac{1}{2}\left(\tilde{\omega}+K_{1} S\right)^{\mathrm{T}} J_{\mathrm{sc}}\left(\tilde{\omega}+K_{1} S\right)+K_{\mathrm{p}} \operatorname{tr}(A-A \tilde{R})+\frac{1}{2} \tilde{\gamma}^{\mathrm{T}} Q \tilde{\gamma}+\frac{1}{2} \tilde{d}^{\mathrm{T}} D \tilde{d}
$$

is positive definite, that is, $V$ is nonnegative, and $V=0$ if and only if $\tilde{\omega}=0, \tilde{R}=I, \tilde{\gamma}=0$, and $\tilde{d}=0$.

Proof. It follows from statement 2 of Lemma 4 that $\operatorname{tr}(A-A \tilde{R})$ is nonnegative. Hence $V$ is nonnegative. Now suppose that $V=0$. Then, $\tilde{\omega}+K_{1} S=0, \tilde{\gamma}=0$, and $\tilde{d}=0$, and it follows from statement 3 of Lemma 4 that $\tilde{R}=I$, and thus $S=0$. Therefore, $\tilde{\omega}=0$.

Note that (43), which we intend to use as a Lyapunov function, is not positive in all the states, namely, it makes no use of the relative angular velocity of the gyro wheels or the gimbals since gyro wheel-speed regulation and gimbal-speed regulation are not goals of the control objective.

Theorem 4. Let $K_{\mathrm{p}}$ be a positive number, let $K_{\mathrm{v}} \in \mathbb{R}^{3 \times 3}, K_{1} \in \mathbb{R}^{3 \times 3}, Q \in \mathbb{R}^{6 \times 6}$, and $D \in \mathbb{R}^{n_{d} \times n_{d}}$ be positive definite matrices, assume that $A_{d}^{\mathrm{T}} D+D A_{d}$ is negative semidefinite, let $A=\operatorname{diag}\left(a_{1}, a_{2}, a_{3}\right)$ be a diagonal positive-definite matrix, define $S$ and $V$ as in Theorem 1, and let $\hat{\gamma}$ and $\hat{d}$ satisfy

$$
\dot{\hat{\gamma}}=Q^{-1}\left[L^{\mathrm{T}}(\omega) \omega^{\times}+L^{\mathrm{T}}\left(K_{1} \dot{S}+\tilde{\omega} \times \omega-\tilde{R}^{\mathrm{T}} \dot{\omega}_{\mathrm{d}}\right)\right]\left(\tilde{\omega}+K_{1} S\right)
$$

where

$$
\dot{S}=\sum_{i=1}^{3} a_{i}\left[\left(\tilde{R}^{\mathrm{T}} e_{i}\right) \times \tilde{\omega}\right] \times e_{i}
$$

and

$$
\begin{aligned}
\dot{\hat{d}} & =A_{d} \hat{d}+D^{-1} C_{d}^{\mathrm{T}}\left(\tilde{\omega}+K_{1} S\right), \\
\hat{\tau}_{\text {dist }} & =C_{d} \hat{d}
\end{aligned}
$$

so that $\hat{\tau}_{\text {dist }}$ is the disturbance torque estimator. Furthermore, consider the control law

$$
u=Y^{-1}\left(v_{1}+v_{2}+v_{3}\right)
$$

where

$$
v_{1} \triangleq-\left(\hat{J}_{\mathrm{sc}} \omega+\sum_{i=1}^{3} \beta_{i} \omega_{\mathrm{W}_{i}}\right) \times \omega-\hat{J}_{\mathrm{sc}}\left(K_{1} \dot{S}+\tilde{\omega} \times \omega-\tilde{R}^{\mathrm{T}} \dot{\omega}_{\mathrm{d}}\right)
$$




$$
v_{2} \triangleq-\hat{\tau}_{\text {dist }}
$$

and

$$
v_{3} \triangleq-K_{\mathrm{v}}\left(\tilde{\omega}+K_{1} S\right)-K_{\mathrm{p}} S
$$

Assume that $Y$ is nonsingular. Then,

$$
\dot{V}(\tilde{\omega}, \tilde{R}, \tilde{\gamma}, \tilde{d})=-\left(\tilde{\omega}+K_{1} S\right)^{\mathrm{T}} K_{\mathrm{v}}\left(\tilde{\omega}+K_{1} S\right)-K_{\mathrm{p}} S^{\mathrm{T}} K_{1} S+\frac{1}{2} \tilde{d}^{\mathrm{T}}\left(A_{d}^{\mathrm{T}} D+D A_{d}\right) \tilde{d},
$$

along the trajectories of (36), is negative semidefinite.

Proof.

$$
\begin{aligned}
& \dot{V}(\tilde{\omega}, \tilde{R}, \tilde{\gamma}, \tilde{d})=\left(\tilde{\omega}+K_{1} S\right)^{\mathrm{T}}\left(J_{\mathrm{sc}} \dot{\tilde{\omega}}+J_{\mathrm{sc}} K_{1} \dot{S}\right)-K_{\mathrm{p}} \operatorname{tr} A \dot{\tilde{R}}-\tilde{\gamma}^{\mathrm{T}} Q \dot{\hat{\gamma}}+\tilde{d}^{\mathrm{T}} D \dot{\tilde{d}} \\
& =\left(\tilde{\omega}+K_{1} S\right)^{\mathrm{T}}\left[\left(J_{\mathrm{sc}} \omega+\sum_{i=1}^{3} \beta_{i} \omega_{\mathrm{W}_{i}}\right) \times \omega+J_{\mathrm{sc}}\left(\tilde{\omega} \times \omega-\tilde{R}^{\mathrm{T}} \dot{\omega}_{\mathrm{d}}\right)+Y u+\tau_{\mathrm{dist}}+J_{\mathrm{sc}} K_{1} \dot{S}\right] \\
& +K_{\mathrm{p}} \tilde{\omega}^{\mathrm{T}} S-\tilde{\gamma}^{\mathrm{T}} Q \dot{\hat{\gamma}}+\tilde{d}^{\mathrm{T}} D \dot{\tilde{d}} \\
& =\left(\tilde{\omega}+K_{1} S\right)^{\mathrm{T}}\left[\left(J_{\mathrm{sc}} \omega+\sum_{i=1}^{3} \beta_{i} \omega_{\mathrm{W}_{i}}\right) \times \omega+J_{\mathrm{sc}}\left(K_{1} \dot{S}+\tilde{\omega} \times \omega-\tilde{R}^{\mathrm{T}} \dot{\omega}_{\mathrm{d}}\right)+v_{1}+v_{2}+v_{3}+\tau_{\text {dist }}\right] \\
& +K_{\mathrm{p}} \tilde{\omega}^{\mathrm{T}} S-\tilde{\gamma}^{\mathrm{T}} Q \dot{\hat{\gamma}}+\tilde{d}^{\mathrm{T}} D \dot{\tilde{d}} \\
& =\left(\tilde{\omega}+K_{1} S\right)^{\mathrm{T}}\left[\left(\tilde{J}_{\mathrm{sc}} \omega\right) \times \omega+\tilde{J}_{\mathrm{sc}}\left(K_{1} \dot{S}+\tilde{\omega} \times \omega-\tilde{R}^{\mathrm{T}} \dot{\omega}_{\mathrm{d}}\right)\right] \\
& +\left(\tilde{\omega}+K_{1} S\right)^{\mathrm{T}} \tilde{\tau}_{\text {dist }}-\left(\tilde{\omega}+K_{1} S\right)^{\mathrm{T}} K_{\mathrm{v}}\left(\tilde{\omega}+K_{1} S\right)-K_{\mathrm{p}}\left(\tilde{\omega}+K_{1} S\right)^{\mathrm{T}} S \\
& +K_{\mathrm{p}} \tilde{\omega}^{\mathrm{T}} S-\tilde{\gamma}^{\mathrm{T}} Q \dot{\hat{\gamma}}+\tilde{d}^{\mathrm{T}} D \dot{\tilde{d}} \\
& =\left(\tilde{\omega}+K_{1} S\right)^{\mathrm{T}}\left[L(\omega) \tilde{\gamma} \times \omega+L\left(K_{1} \dot{S}+\tilde{\omega} \times \omega-\tilde{R}^{\mathrm{T}} \dot{\omega}_{\mathrm{d}}\right) \tilde{\gamma}\right] \\
& -\left(\tilde{\omega}+K_{1} S\right)^{\mathrm{T}} K_{\mathrm{v}}\left(\tilde{\omega}+K_{1} S\right)-K_{\mathrm{p}} S^{\mathrm{T}} K_{1} S-\tilde{\gamma}^{\mathrm{T}} Q \dot{\hat{\gamma}} \\
& +\tilde{d}^{\mathrm{T}} C_{d}^{\mathrm{T}}\left(\tilde{\omega}+K_{1} S\right)+\tilde{d}^{\mathrm{T}} D\left[A_{d} \tilde{d}-D^{-1} C_{d}^{\mathrm{T}}\left(\tilde{\omega}+K_{1} S\right)\right] \\
& =-\left(\tilde{\omega}+K_{1} S\right)^{\mathrm{T}} K_{\mathrm{v}}\left(\tilde{\omega}+K_{1} S\right)-K_{\mathrm{p}} S^{\mathrm{T}} K_{1} S-\tilde{\gamma}^{\mathrm{T}} Q \dot{\hat{\gamma}} \\
& +\left(\tilde{\omega}+K_{1} S\right)^{\mathrm{T}}\left[-\omega^{\times} L(\omega)+L\left(K_{1} \dot{S}+\tilde{\omega} \times \omega-\tilde{R}^{\mathrm{T}} \dot{\omega}_{\mathrm{d}}\right)\right] \tilde{\gamma} \\
& +\frac{1}{2} \tilde{d}^{\mathrm{T}}\left(A_{d}^{\mathrm{T}} D+D A_{d}\right) \tilde{d} \\
& =-\left(\tilde{\omega}+K_{1} S\right)^{\mathrm{T}} K_{\mathrm{v}}\left(\tilde{\omega}+K_{1} S\right)-K_{\mathrm{p}} S^{\mathrm{T}} K_{1} S \\
& +\tilde{\gamma}^{\mathrm{T}}\left[-Q \dot{\hat{\gamma}}+\left(L^{\mathrm{T}}(\omega) \omega^{\times}+L^{\mathrm{T}}\left(K_{1} \dot{S}+\tilde{\omega} \times \omega-\tilde{R}^{\mathrm{T}} \dot{\omega}_{\mathrm{d}}\right)\right)\left(\tilde{\omega}+K_{1} S\right)\right] \\
& +\frac{1}{2} \tilde{d}^{\mathrm{T}}\left(A_{d}^{\mathrm{T}} D+D A_{d}\right) \tilde{d} \\
& =-\left(\tilde{\omega}+K_{1} S\right)^{\mathrm{T}} K_{\mathrm{v}}\left(\tilde{\omega}+K_{1} S\right)-K_{\mathrm{p}} S^{\mathrm{T}} K_{1} S+\frac{1}{2} \tilde{d}^{\mathrm{T}}\left(A_{d}^{\mathrm{T}} D+D A_{d}\right) \tilde{d} .
\end{aligned}
$$

Future work will complete the proof for almost global stabilization (that is, Lyapunov stability with almost global convergence) of spacecraft tracking using CMGs. The proof will rely on partial stability theory and invariance theorems.

\section{Modification to avoid singularities}

A difficulty encountered with CMGs is that the torque they are able to generate may sometimes lie in a plane perpendicular to the demanded torque. When this condition takes place they are considered to be in a singular state, and gimbal angular velocities that synthesize the requested torque do not exist. Much of the work on CMGs has thus been to develop steering laws, that is, laws that modify the controller requested torque to either avoid these singular states, or steer the controller through them

While Theorems 2 and 4 do not use an explicit steering law to synthesize a desired torque, the matrix $Y$, which is assumed to be invertible in these theorems, plays a similar role and is sometimes not invertible. 
Borrowing ideas from the steering law literature, we therefore examine two practical, albeit approximate, methods for inverting $Y$. Section VI.A discusses a modified version of the pseudoinverse, while section VI.B discusses the singularity-robust (SR) inverse. ${ }^{24}$ Other methods such as the singular-direction avoidance (SDA) inverse ${ }^{22}$ are left for future study.

\section{A. Saturated pseudoinverse method}

We consider taking the saturated pseudoinverse of $Y$. We define the saturated pseudoinverse as

$$
Y^{+s a t}=V \Sigma^{-1_{s a t}} U^{T},
$$

where $U \in \mathbb{R}^{3 \times 3}$ and $V \in \mathbb{R}^{3 \times 3}$ are orthogonal matrices obtained from the singular value decomposition of $Y$, which is given by

$$
Y=U \Sigma V^{\mathrm{T}}
$$

where $\Sigma \in \mathbb{R}^{3 \times 3}$ is a diagonal matrix with singular values of $Y$ on the diagonal,

$$
\Sigma=\left[\begin{array}{ccc}
\sigma_{1} & 0 & 0 \\
0 & \sigma_{2} & 0 \\
0 & 0 & \sigma_{3}
\end{array}\right]
$$

and $\Sigma^{-1_{\text {sat }}}$ is defined as

$$
\Sigma^{-1_{s a t}}=\left[\begin{array}{ccc}
\min \left(\sigma_{1}{ }^{-1}, c_{1}^{-1}\right) & 0 & 0 \\
0 & \min \left(\sigma_{2}{ }^{-1}, c_{1}^{-1}\right) & 0 \\
0 & 0 & \min \left(\sigma_{3}{ }^{-1}, c_{1}^{-1}\right)
\end{array}\right],
$$

where $c_{1}>0$ is a saturation limit. Note that unlike the standard pseudoinverse of a matrix approaching rank deficiency, whose matrix norm grows quite large until the matrix is within some tolerance of singularity and then becomes singular, the saturated pseudoinverse is always full rank and is saturated at $c_{1}^{-1}$.

Using the saturated pseudoinverse, control laws (39) and (48) become

$$
u=-Y^{+s a t}\left(K_{\mathrm{p}} S+K_{\mathrm{v}} \omega\right) .
$$

and

$$
u=Y^{+s a t}\left(v_{1}+v_{2}+v_{3}\right) .
$$

Additionally, if the saturated pseudoinverse based controllers (57), (58) specify inputs with magnitudes larger than $u_{\max }$, let

$$
u_{\mathrm{sat}}=u_{\max } \frac{u}{\|u\|} .
$$

We use (59) only when $\Sigma^{-1} \neq \Sigma^{-1_{s a t}}$ in order to prevent $\dot{u}$ from becoming arbitrarily large. Note that (57), (58) reduce to (39),(48) when $Y$ is nonsingular.

\section{B. Singularity Robust inverse method}

One technique used in steering laws is the singularity robust (SR) inverse. ${ }^{25}$ The idea behind the SR inverse is that it trades off between introducing torque errors in the vicinity of a singularity and the feasibility of the solution - feasibility indicating that the gimbal angular velocities remain bounded, unlike the case of the Moore-Penrose inverse.

The SR inverse is derived from the following optimization problem,

$$
\text { minimize } e^{\mathrm{T}} W e
$$


where $e=\left[\begin{array}{ll}\tau-Y u & u\end{array}\right]^{\mathrm{T}}, \tau$ is the desired torque in a steering law formulation of the CMG problem, and $W=\operatorname{diag}\left(W_{1}, W_{2}\right)$ is a block diagonal weight matrix.

The SR inverse is thus given by

$$
Y^{\#}=W_{2}^{-1} Y^{\mathrm{T}}\left(Y W_{2} Y^{\mathrm{T}}+W_{1}^{-1}\right)
$$

Note that different values of $W_{1}$ and $W_{2}$ yield different SR inverses and that by selecting $W_{1}=0$ and $W_{2}=I$ one recovers the Moore-Penrose inverse.

We consider the SR inverse for the inversion of $Y$ in (39) and (48). Since the SR inverse introduces error into the inversion, we test both control laws ability to compensate for this disturbance. The control laws thus become

$$
u=-Y^{\#}\left(K_{\mathrm{p}} S+K_{\mathrm{v}} \omega\right)
$$

and

$$
u=Y^{\#}\left(v_{1}+v_{2}+v_{3}\right) .
$$

In the simulations below we do not modify the weight matrices $W_{1}$ and $W_{2}$ based on the distance of $Y$ from singularity.

\section{Simulation}

Simulation results are used to illustrate the effectiveness of controllers (57), (58), (62) and (63) in controlling the spacecraft attitude and angular velocity using CMG actuators. To simulate the rest-to-rest, motion-to-rest and spin maneuvers, the following spacecraft parameters are assumed. The bus inertia matrix $J_{\mathrm{b}}$ is given by

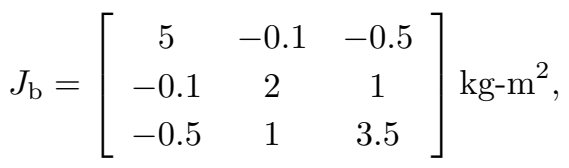

with principal moments of inertia $1.4947,3.7997$, and $5.2056 \mathrm{~kg}-\mathrm{m}^{2} . J_{\mathrm{b}}$ is unknown to the controller. The axes of rotation of the three CMGs are aligned with the spacecraft body-fixed frame unit vectors, and we assume that the gyro wheel inertias in their home position with respect to their own centers are given by $J_{\mathrm{w}_{1}}=\operatorname{diag}(0.3,0.3,0.3) \mathrm{kg}-\mathrm{m}^{2}, J_{\mathrm{w}_{2}}=\operatorname{diag}(0.3,0.3,0.3) \mathrm{kg}-\mathrm{m}^{2}$, and $J_{\mathrm{w}_{3}}=\operatorname{diag}(0.3,0.3,0.3) \mathrm{kg}-\mathrm{m}^{2}$. The gyro wheels are assumed to be running at a constant speed of $20 \mathrm{rad} / \mathrm{sec}$. Let $K_{\mathrm{v}}=I_{3}$, and let $K_{\mathrm{p}}$ be given by

$$
K_{\mathrm{p}}=\frac{\alpha}{\operatorname{tr} A}
$$

Illustrative rest-to-rest, motion-to-rest and spin maneuvers are considered below.

\section{A. Rest-to-Rest Maneuver using Control Laws (57) and (62)}

We use controller (57) for a rest-to-rest maneuver, where the objective is to bring the spacecraft from the initial attitude $R_{0}=I_{3}$ to the desired final orientation

$$
R_{\mathrm{d}}=\left[\begin{array}{ccc}
0.4536 & 0.8912 & 0 \\
-0.8912 & 0.4536 & 0 \\
0 & 0 & 1.0000
\end{array}\right]
$$

which represents a rotation of 1.1 radians about the $z$-axis. We assume that the gimbals are initially at rest relative to the spacecraft, that is,

$$
u(0)=\left[\begin{array}{lll}
0 & 0 & 0
\end{array}\right]^{\mathrm{T}} \mathrm{rad} / \mathrm{sec} .
$$


The initial gimbal angles are $\phi_{1}=0 \mathrm{rad}, \phi_{2}=0.419 \mathrm{rad}, \phi_{3}=3.14 \mathrm{rad}$. Let $c_{1}=2$ and $u_{\max }=3$.

Figures 1(a)-(f) show, respectively, the attitude error, angular velocity components, gimbal angles, gimbal angular velocity control inputs $(u)$, gimbal angular accelerations $(\dot{u})$ and singular values of $Y$. The spacecraft attitude and angular velocity components are brought close to the desired values in about 10 sec. It can be seen from Figure 1(e) that the control input derivatives decrease to zero in about 0.5 sec. Figure 1(f) confirms that the controller is successfully able to avoid singularities during this maneuver.

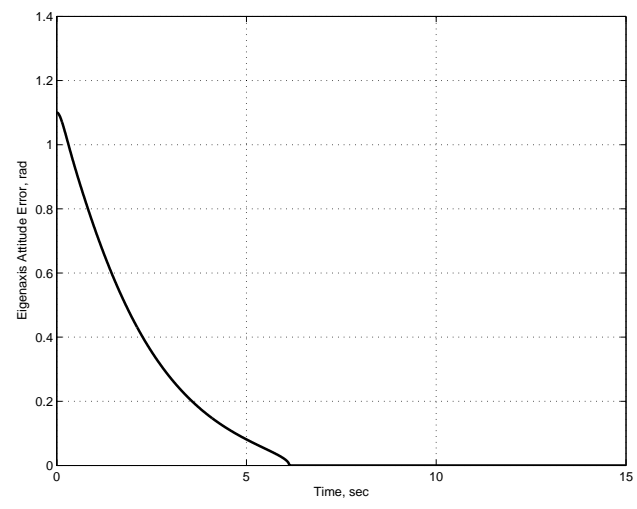

(a) Eigenaxis attitude error.



(c) Gimbal Angles.

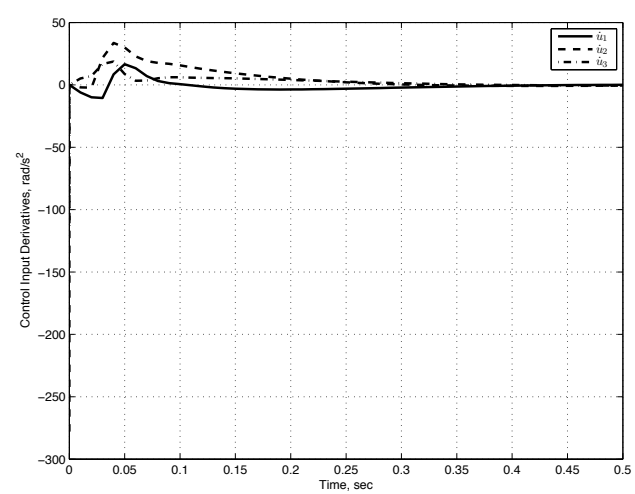

(e) Control input derivatives of the gimbals.

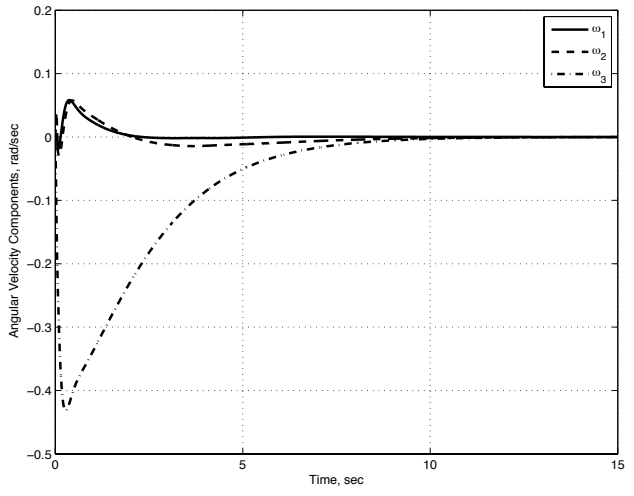

(b) Spacecraft angular-velocity components.

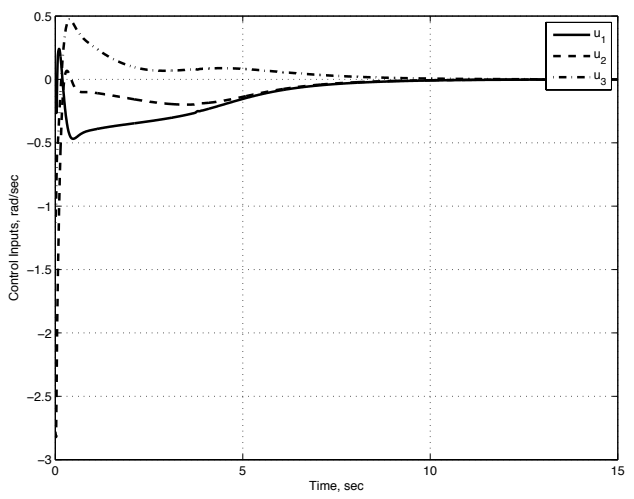

(d) Control input to the gimbals.

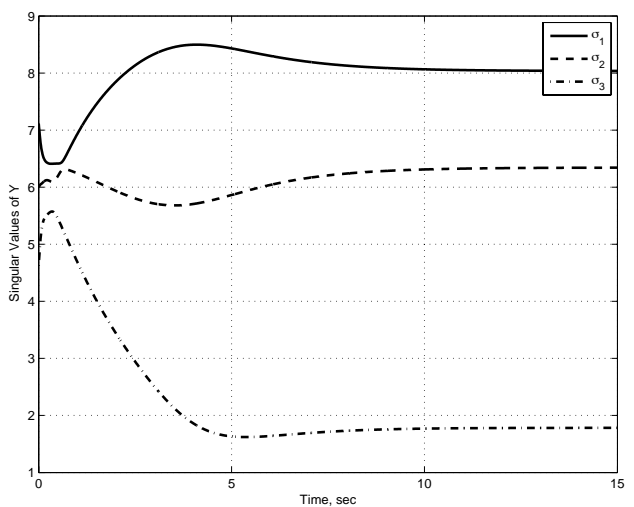

(f) Singular values of the matrix $Y$.

Figure 1: Rest-to-Rest maneuver with CMGs using control law (57).

We now use controller (62) for the same maneuver. Let $W_{1}=\operatorname{diag}(7,8,9)$ and $W_{2}=\operatorname{diag}(14,15,6)$. The initial gimbal angles in this case are $\phi_{1}=0 \mathrm{rad}, \phi_{2}=0.897 \mathrm{rad}, \phi_{3}=1.744 \mathrm{rad}$. All other initial conditions are the same as in the previous maneuver.

Figures 2(a)-(f) show, respectively, the attitude error, angular velocity components, gimbal angles, gimbal angular velocity control inputs $(u)$, gimbal angular accelerations $(\dot{u})$ and singular values of $Y$. The spacecraft 
attitude and angular velocity components are brought close to the desired values in about 10 sec. It can be seen from Figure 2(e) that the control input derivatives decrease to zero in about 1 sec. Figure 2(f) confirms that the controller is successfully able to avoid singularities during this maneuver.

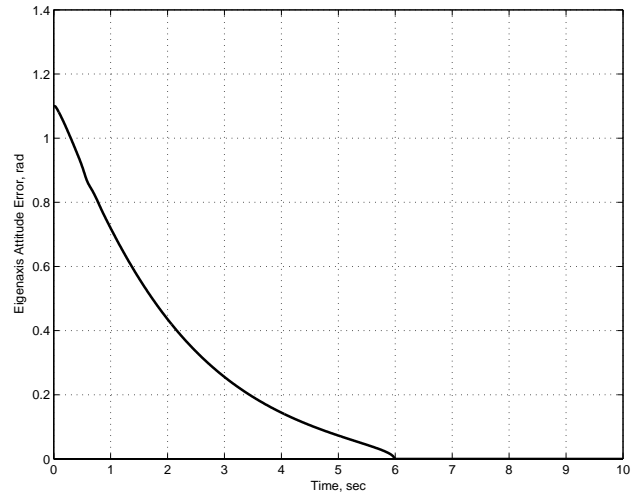

(a) Eigenaxis attitude error.

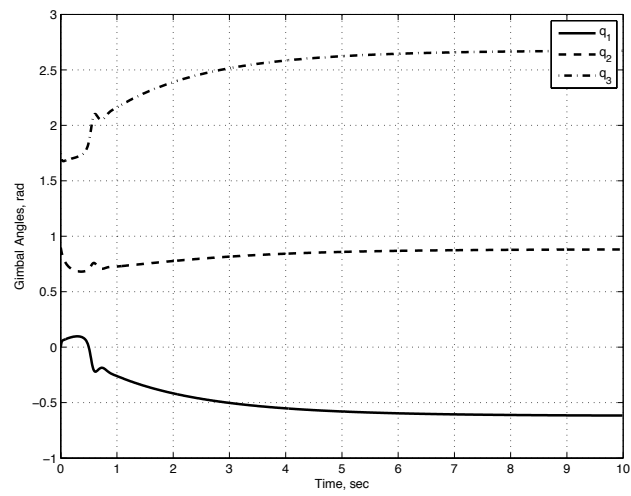

(c) Gimbal Angles.

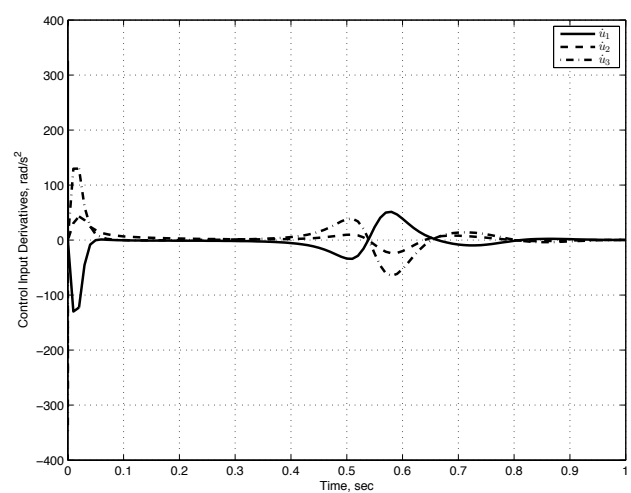

(e) Control input derivatives of the gimbals.

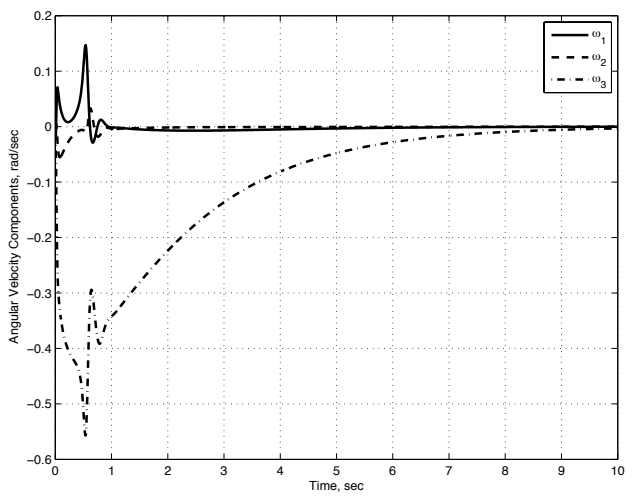

(b) Spacecraft angular-velocity components.

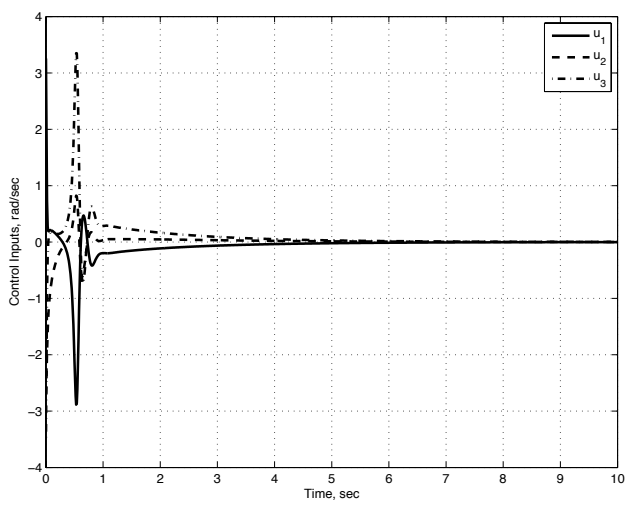

(d) Control input to the gimbals.

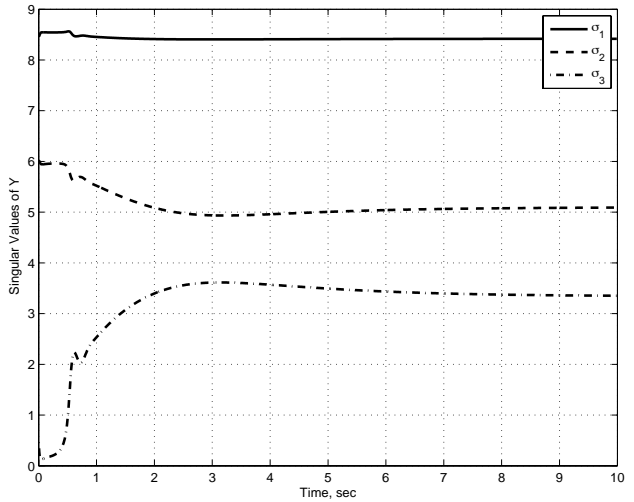

(f) Singular values of the matrix $Y$.

Figure 2: Rest-to-Rest maneuver with CMGs using control law (62).

\section{B. Motion-to-Rest Maneuver using Control Laws (57) and (62)}

We now consider a motion-to-rest maneuver, where the spacecraft is initially spinning with an angular velocity

$$
\omega_{0}=\left[\begin{array}{lll}
0.5 & -0.5 & -0.5
\end{array}\right]^{\mathrm{T}} \mathrm{rad} / \mathrm{sec},
$$


and initial attitude $R_{\mathrm{d}}(0)=I_{3}$, where the objective is to bring the spacecraft to rest at the desired final orientation $R_{\mathrm{d}}$ as given above. The initial gimbal angles are $\phi_{1}=0 \mathrm{rad}, \phi_{2}=0.419 \mathrm{rad}, \phi_{3}=3.14 \mathrm{rad}$. Let $c_{1}=1$ and $u_{\max }=15$.

Figures 3(a)-(f) show, respectively, the attitude error, angular velocity components, gimbal angles, gimbal angular velocity control inputs $(u)$, gimbal angular accelerations $(\dot{u})$ and singular values of $Y$. The spacecraft attitude and angular velocity components are brought close to the desired values in about 5 sec. It can be seen from Figure 3(e) that the control input derivatives decrease to zero in about 0.8 sec. Figure 3(f) confirms that the controller is successfully able to avoid singularities during this maneuver.

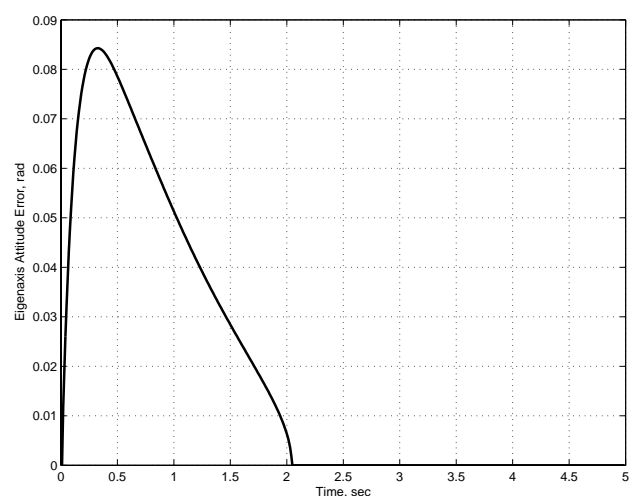

(a) Eigenaxis attitude error.

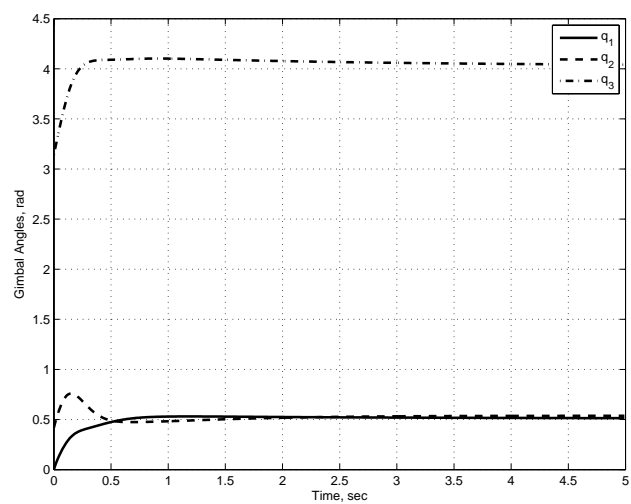

(c) Gimbal Angles.

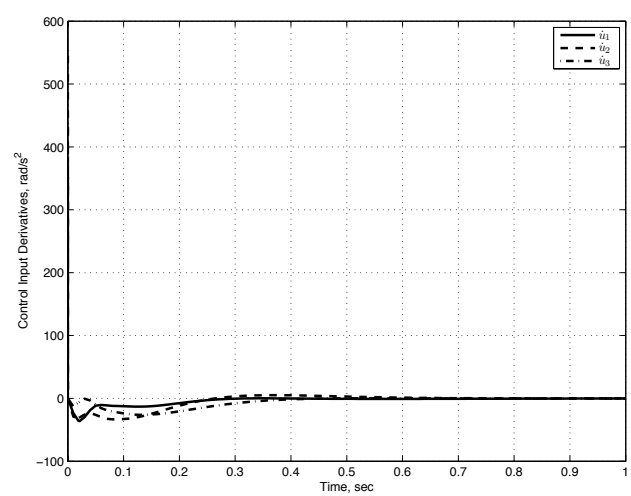

(e) Control input derivatives of the gimbals.



(b) Spacecraft angular-velocity components.

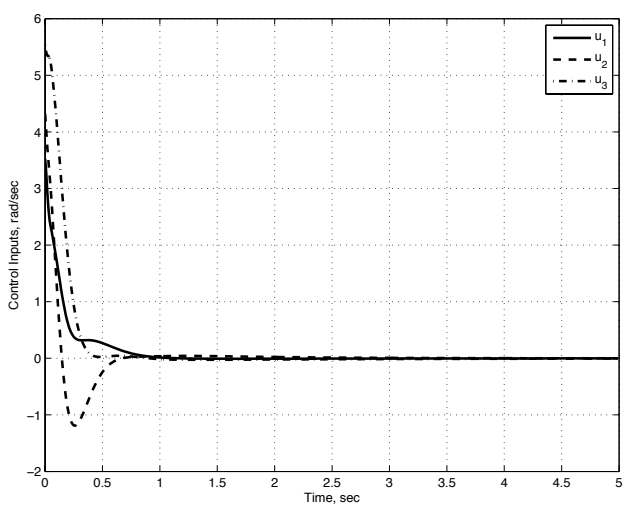

(d) Control input to the gimbals.

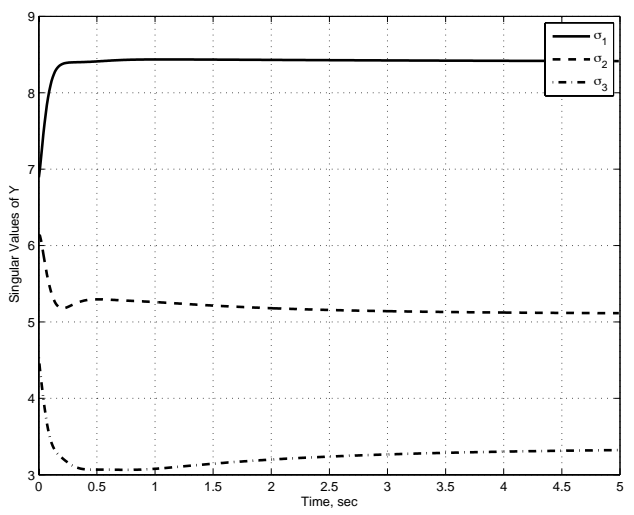

(f) Singular values of the matrix $Y$.

Figure 3: Motion-to-Rest maneuver with CMGs using control law (57).

We now use controller (62) for the same maneuver. Let $W_{1}=\operatorname{diag}(7,8,9)$ and $W_{2}=\operatorname{diag}(14,15,6)$. The initial gimbal angles in this case are $\phi_{1}=0 \mathrm{rad}, \phi_{2}=0.897 \mathrm{rad}, \phi_{3}=1.744 \mathrm{rad}$. All other initial conditions are the same as in the previous maneuver. 
Figures 4(a)-(f) show, respectively, the attitude error, angular velocity components, gimbal angles, gimbal angular velocity control inputs $(u)$, gimbal angular accelerations $(\dot{u})$ and singular values of $Y$. The spacecraft attitude and angular velocity components are brought close to the desired values in about 2.5 sec. It can be seen from Figure 4(e) that the control input derivatives decrease to zero in about 0.3 sec. Figure $4(\mathrm{f})$ confirms that the controller is successfully able to avoid singularities during this maneuver.

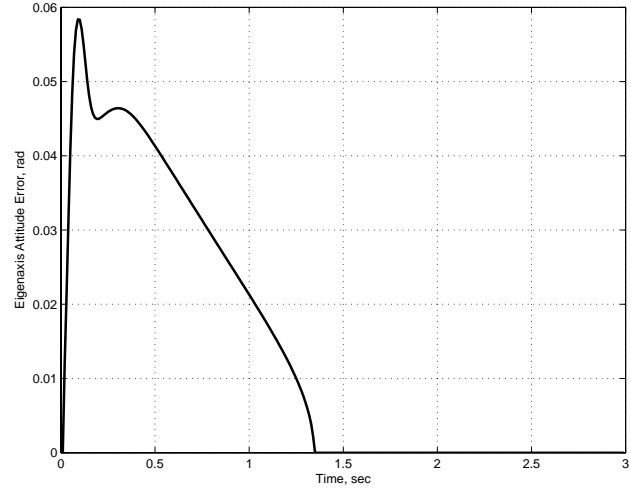

(a) Eigenaxis attitude error.



(c) Gimbal Angles.

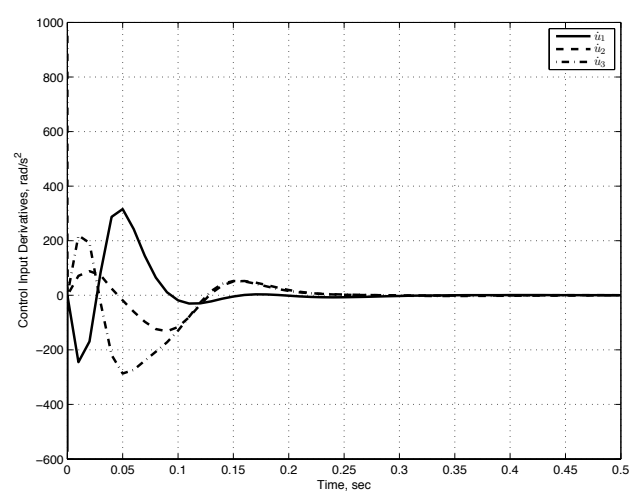

(e) Control input derivatives of the gimbals.

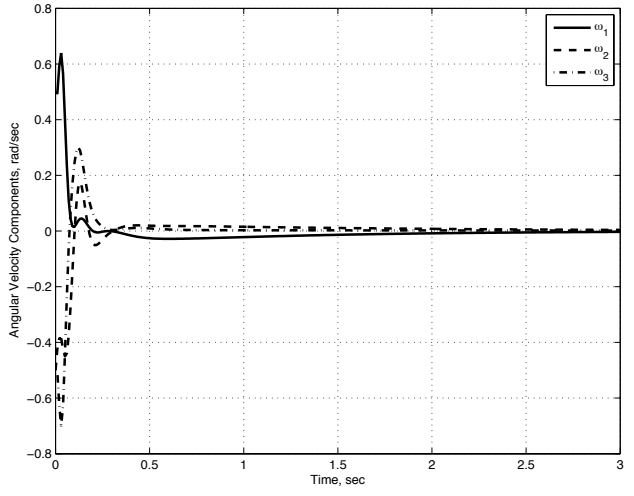

(b) Spacecraft angular-velocity components.

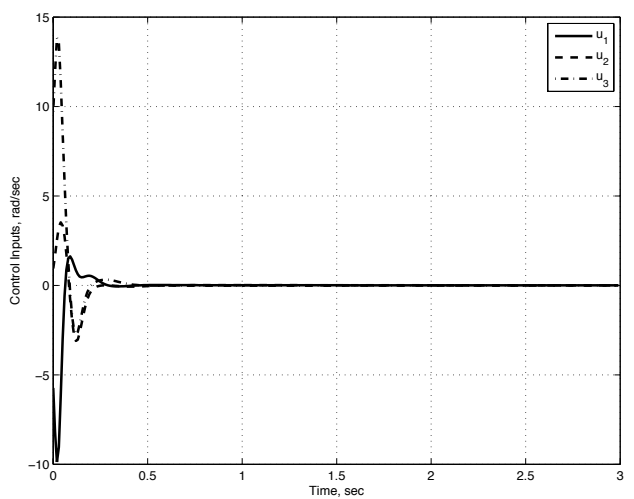

(d) Control input to the gimbals.

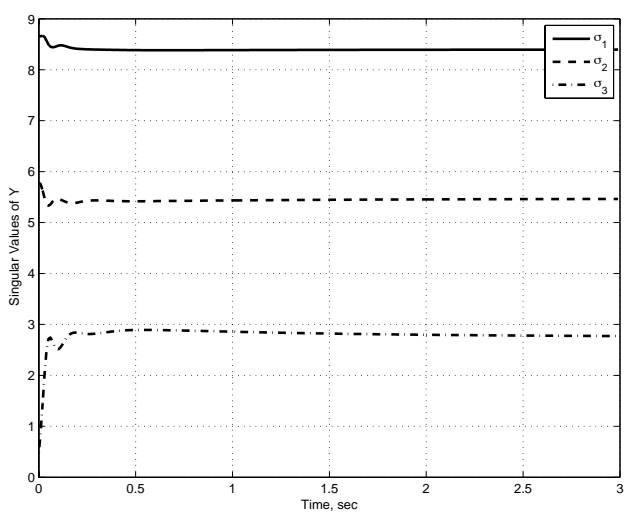

(f) Singular values of the matrix $Y$.

Figure 4: Motion-to-Rest maneuver with CMGs using control law (62). 


\section{Rest-to-Rest Maneuver using Control Laws (58) and (63)}

We use controller (58) for a rest-to-rest maneuver, where the objective is to bring the spacecraft from the initial attitude $R_{0}=I_{3}$ to the desired final orientation

$$
R_{\mathrm{d}}=\left[\begin{array}{ccc}
0.0707 & 0.9975 & 0 \\
-0.9975 & 0.0707 & 0 \\
0 & 0 & 1.0000
\end{array}\right]
$$

which represents a rotation of 1.5 radians about the $z$-axis. We assume that the gimbals are initially at rest relative to the spacecraft, that is,

$$
u(0)=\left[\begin{array}{lll}
0 & 0 & 0
\end{array}\right]^{\mathrm{T}} \mathrm{rad} / \mathrm{sec} .
$$

The initial gimbal angles are $\phi_{1}=0 \mathrm{rad}, \phi_{2}=0.419 \mathrm{rad}, \phi_{3}=3.14 \mathrm{rad}$. The parameters of controller (58) are chosen to be $K_{1}=I_{3}, \alpha=1, A=\operatorname{diag}(1,2,3), D=I_{3}$, and $Q=I_{6}$. Let $c_{1}=1$ and $u_{\max }=1$.

Figures 5(a)-(g) show, respectively, the attitude error, angular velocity components, gimbal angles, gimbal angular velocity control inputs $(u)$, gimbal angular accelerations $(\dot{u})$, singular values of $Y$ and inertia estimate errors. The spacecraft attitude and angular velocity components are brought close to the desired values in about 15 sec. It can be seen from Figure 5(e) that the control input derivatives decrease to zero in about 6 sec. Figure 5(f) confirms that the controller is successfully able to pass through singularities during this maneuver.

We now use controller (63) for the same maneuver. Let $W_{1}=\operatorname{diag}(7,8,9)$ and $W_{2}=\operatorname{diag}(14,15,6)$. The initial gimbal angles are $\phi_{1}=0 \mathrm{rad}, \phi_{2}=0.897 \mathrm{rad}, \phi_{3}=1.744 \mathrm{rad}$. All other initial conditions and controller parameters are the same as in the previous maneuver.

Figures 6(a)-(g) show, respectively, the attitude error, angular velocity components, gimbal angles, gimbal angular velocity control inputs $(u)$, gimbal angular accelerations $(\dot{u})$, singular values of $Y$ and inertia estimate errors. The spacecraft attitude and angular velocity components are brought close to the desired values in about 15 sec. It can be seen from Figure 6(e) that the control input derivatives decrease to zero in about 5 sec. Figure $6(\mathrm{f})$ confirms that the controller is successfully able to pass through singularities during this maneuver.

\section{Motion-to-Rest Maneuver using Control Laws (58) and (63)}

We now consider a motion-to-rest maneuver, where the spacecraft is initially spinning with an angular velocity

$$
\omega_{0}=\left[\begin{array}{lll}
0.5 & -1 & -0.5
\end{array}\right]^{\mathrm{T}} \mathrm{rad} / \mathrm{sec},
$$

and initial attitude $R_{\mathrm{d}}(0)=I_{3}$, where the objective is to bring the spacecraft to rest at the desired final orientation $R_{\mathrm{d}}$ as given above. Likewise, the controller parameters are as given in the previous section. The initial gimbal angles are $\phi_{1}=0 \mathrm{rad}, \phi_{2}=0.419 \mathrm{rad}, \phi_{3}=3.14 \mathrm{rad}$. Let $c_{1}=1$ and $u_{\max }=1$.

Figures 7(a)-(g) show, respectively, the attitude error, angular velocity components, gimbal angles, gimbal angular velocity control inputs $(u)$, gimbal angular accelerations $(\dot{u})$, singular values of $Y$ and inertia estimate errors. The spacecraft attitude and angular velocity components are brought close to the desired values in about 25 sec. It can be seen from Figure $7(\mathrm{e})$ that the control input derivatives decrease to zero in about 0.1 sec. Figure $7(f)$ confirms that the controller is successfully able to pass through singularities during this maneuver.

We now use controller (63) for the same maneuver. Let $W_{1}=\operatorname{diag}(7,8,9)$ and $W_{2}=\operatorname{diag}(140,150,60)$. The initial gimbal angles are $\phi_{1}=0 \mathrm{rad}, \phi_{2}=0.897 \mathrm{rad}, \phi_{3}=1.744 \mathrm{rad}$. All other initial conditions and controller parameters are the same as in the previous maneuver.

Figures 8(a)-(g) show, respectively, the attitude error, angular velocity components, gimbal angles, gimbal angular velocity control inputs $(u)$, gimbal angular accelerations $(\dot{u})$, singular values of $Y$ and inertia estimate errors. The spacecraft attitude and angular velocity components are brought close to the desired values in about 20 sec. It can be seen from Figure 8(e) that the control input derivatives decrease to zero in about 10 sec. Figure $8(\mathrm{f})$ confirms that the controller is successfully able to pass through singularities during this maneuver. 


\section{E. Spin Maneuver using Control Law (63)}

We use controller (63) for a spin maneuver, where the objective is to bring the spacecraft from rest to a spin of

$$
\omega_{\mathrm{d}}=\left[\begin{array}{lll}
0.005 & -0.005 & 0.003
\end{array}\right]^{\mathrm{T}} \mathrm{rad} / \mathrm{sec}
$$

We assume that the gimbals are initially at rest relative to the spacecraft, that is,

$$
u(0)=\left[\begin{array}{lll}
0 & 0 & 0
\end{array}\right]^{\mathrm{T}} \mathrm{rad} / \mathrm{sec} .
$$

Let $W_{1}=\operatorname{diag}(7,8,9)$ and $W_{2}=\operatorname{diag}(14,15,6)$. The initial gimbal angles are $\phi_{1}=0 \mathrm{rad}, \phi_{2}=0.897$ $\mathrm{rad}, \phi_{3}=1.744 \mathrm{rad}$. All other controller parameters are the same as in the previous section.

Figures 9(a)-(g) show, respectively, the attitude error, angular velocity components, gimbal angles, gimbal angular velocity control inputs $(u)$, gimbal angular accelerations $(\dot{u})$, singular values of $Y$ and inertia estimate errors. The spacecraft attitude and angular velocity components are brought close to the desired values in about 70 sec. It can be seen from Figure 9(e) that the control input derivatives decrease to zero in about 20 sec. Figure $9(\mathrm{f})$ confirms that the controller is successfully able to avoid singularities during this maneuver.

\section{Conclusion}

We extended the control laws of ref. ${ }^{1}$ to the case of control moment gyroscope actuation with gimbal velocity commands. We carefully derived the equations of motion of a spacecraft with three linearly independent CMG actuators with spherical gyro wheels, whose gimbal axes of rotation are not necessarily aligned with the principal axes of the spacecraft bus, do not necessarily pass through the spacecraft's center of mass, and are not necessarily mass balanced in order to preserve the location of the spacecraft's center of mass. We do not require knowledge of the spacecraft's inertia, only the inertia of the gyro wheels. These results have practical advantages relative to previous controllers that 1) require exact or approximate inertia information or 2) are based on attitude parameterizations such as quaternions that require discontinuous control laws or fail to be physically consistent (that is, specify different control torques for the same physical orientation).

We discussed two singularity avoidance methods in this paper, namely, the saturated pseudoinverse and the singularity robust (SR) inverse. We simulated the controllers for rest-to-rest, motion-to-rest, and spin maneuvers that pass through CMG singularities using both methods. We found that the spacecraft achieves the desired orientation and spin.

\section{References}

${ }^{1}$ A. Sanyal, A. Fosbury, N. Chaturvedi, and D. S. Bernstein, "Inertia-Free Spacecraft Attitude Tracking with Disturbance Rejection and Almost Global Stabilization," AIAA J. Guid. Contr. Dyn., Vol. 32, pp. 1167-1178, 2009.

${ }^{2}$ Weiss, A., Yang, X., Kolmanovsky I., and Bernstein D.S., "Inertia-Free Spacecraft Attitude Control with Reaction-Wheel Actuation," AIAA Guidance, Navigation, and Control Conference, AIAA-2010-8297, 2010.

${ }^{3}$ Ahmed, J., Coppola, V. T., and Bernstein, D. S., "Asymptotic Tracking of Spacecraft Attitude Motion with Inertia Matrix Identification," AIAA Journal of Guidance, Control, and Dynamics, Vol. 21, pp. 684-691, 1998.

${ }^{4}$ Chaturvedi N., Sanyal A., and McClamroch, N. H., "Rigid Body Attitude Control: Using rotation matrices for continuous, singularity-free control laws," IEEE Control Systems Magazine, Vol. 31(3), pp. 30-51, 2011.

${ }^{5}$ Wie, B., and Barba, P. M., "Quaternion Feedback for Spacecraft Large Angle Maneuvers," AIAA Journal of Guidance, Control, and Dynamics, Vol. 8, pp. 360-365, 1985.

${ }^{6}$ Joshi, S. M., Kelkar, A. G., and Wen, J. T., "Robust attitude stabilization using nonlinear quaternion feedback," IEEE Transactions on Automatic Control, Vol. 40, pp. 1148-1161, 1995.

${ }^{7}$ Bhat, S. P., and Bernstein, D. S., "A Topological Obstruction to Continuous Global Stabilization of Rotational Motion and the Unwinding Phenomenon," Systems and Control Letters, Vol. 39, pp. 63-70, 2000.

${ }^{8}$ Cortes, J., "Discontinuous Dynamic Systems," IEEE Control Systems Magazine, Vol. 28, pp. 36-71, June 2008.

${ }^{9}$ Crassidis, J. L., Vadali, S. R., and Markley, F. L., "Optimal Variable-Structure Control Tracking of Spacecraft Maneuvers," AIAA Journal of Guidance, Control, and Dynamics, Vol. 23, pp. 564-566, 2000.

${ }^{10}$ Mayhew, C. G., Sanfelice, R. G., and Teel, A. R., "Robust Global Asymptotic Stabilization of a 6-DOF Rigid Body by Quaternion-based Hybrid Feedback," Proc. IEEE CDC, pp. 1094-1099, 2009.

${ }^{11}$ Wie, B., Space Vehicle Dynamics and Control, AIAA, 2008.

${ }^{12}$ Wie, B., "Singularity Analysis and Visualization for Single-Gimbal Control Moment Gyro Systems," Journal of Guidance, Control, and Dynamics, vol. 27, no. 2, pp. 271-282, 2004. 
${ }^{13}$ Kurokawa, H., "A Geometry Study of Single Gimbal Control Moment Gyros - Singularity Problem and Steering Law, TR 175, Mechanical Engineering Lab., Tsukuba, Ibaraki, Japan, 1998.

${ }^{14}$ Takada, K., Kojima, H., and Matsuda, N., "Control Moment Gyro Singularity-Avoidance Steering Control Based on Singular-Surface Cost Function," Journal of Guidance, Control, and Dynamics, vol. 33, no. 5, pp. 1442-1450, 2010.

${ }^{15}$ Wie, B., "Singularity Robust Steering Logic for Redundant Single-Gimbal Control Moment Gyros," Journal of Guidance, Control, and Dynamics, vol. 24, no. 5, pp. 865-872, 2001.

${ }^{16}$ Paradiso, J.A., "Global Steering of Single Gimbaled Control Moment Gyroscopes using a Directed Search," Journal of Guidance, Control, and Dynamics, vol. 15, no. 5, pp. 1236-1244, 1992.

${ }^{17}$ Gersh, J., and Peck, M., "Violet: A High-Agility Nanosatellite for Demonstrating Small Control-Moment Gyroscope Prototypes and Steering Laws," AIAA Guidance, Navigation, and Control Conference, Chicago, Illinois, August 10-13, 2009.

${ }^{18}$ Yoon, H., Tsiotras, P., "Singularity Analysis of Variable-Speed Control Moment Gyros," Journal of Guidance, Control, and Dynamics, vol. 27, no. 3, pp. 374-386, 2004.

${ }^{19}$ Hughes, P. C., Spacecraft Attitude Dynamics, Wiley, 1986; reprinted by Dover, 2008, page 17.

${ }^{20}$ Sanyal, A. K., Lee, T., Leok, M., and McClamroch, N. H., "Global Optimal Attitude Estimation Using Uncertainty Ellipsoids," Systems and Control Letters, Vol. 57, pp. 236-245, 2008.

${ }^{21}$ F. Leve, G.A. Boyarko, and N.G. Fitz-Coy, "Precise Torque Mapping for Pico-Satellite Single-Gimbal Control Moment Gyroscopes," Advances in the Astronautical Sciences, Vol. 137, Univelt, Inc., San Diego, CA, 2010, also AAS/AIAA Paper AAS 10-095, 33rd Annual AAS Guidance and Control Conference, Breckenridge, CO, February, 2010.

${ }^{22}$ Meng, T., and Matunaga, S. "Modified Singular-Direction Avoidance Steering for Control Moment Gyros," Journal of Guidance, Control, and Dynamics, vol. 34, no. 6, pp. 1915-1919, 2011.

${ }^{23}$ Pechev, A. "Feedback-Based Steering Law for Control Moment Gyros," Journal of Guidance, Control, and Dynamics, vol. 30, no. 3, pp. 848-855, 2007.

${ }^{24}$ Nakamura, Y., Hanafusa, H., "Inverse Kinematic Solutions with Singularity Robustness for Robot Manipulator Control", Journal of Dynamic Systems, Measurement, and Control, Vol. 108, Sept. 1986, pp. 163-171.

${ }^{25}$ Bedrossian, N.S., Steering Law Design for Redundant Single Gimbal Control Moment Gyro Systems, M.S. Thesis, Massachusetts Inst. of Technology, Cambridge, MA, Aug. 1987. 


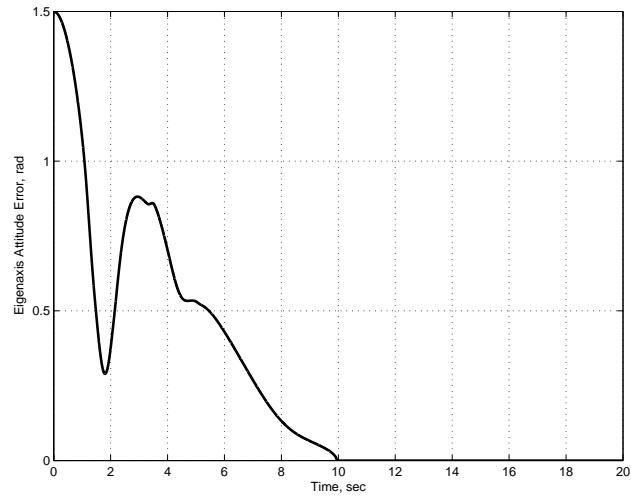

(a) Eigenaxis attitude error.

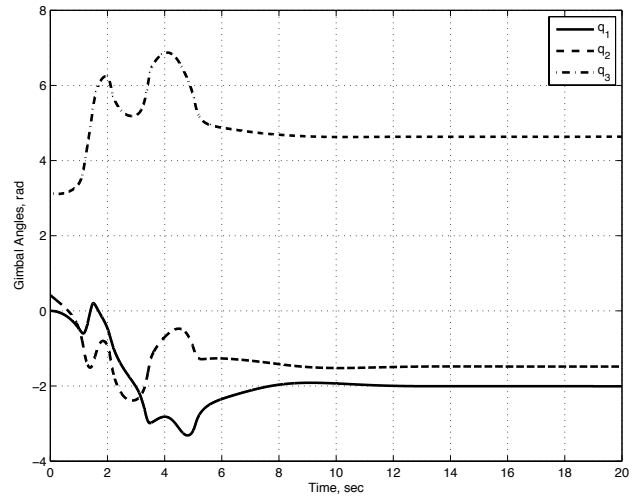

(c) Gimbal Angles.

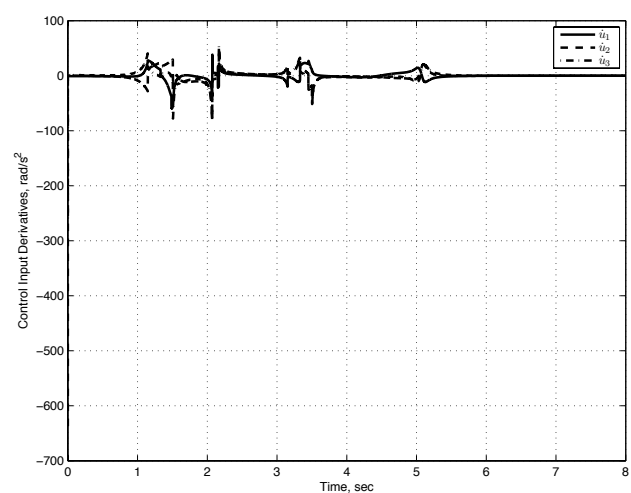

(e) Control input derivatives of the gimbals.

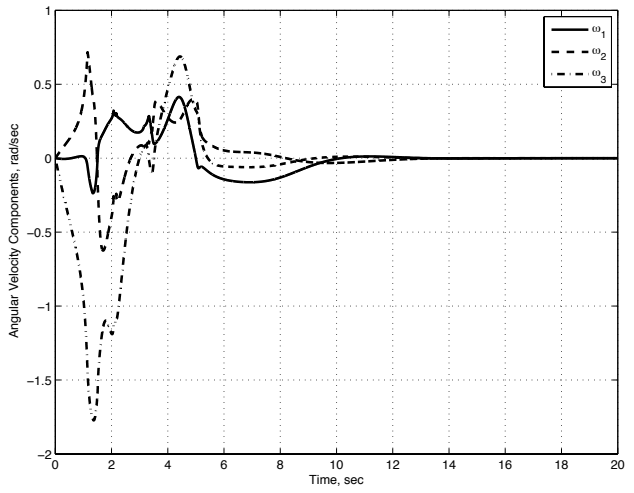

(b) Spacecraft angular-velocity components.

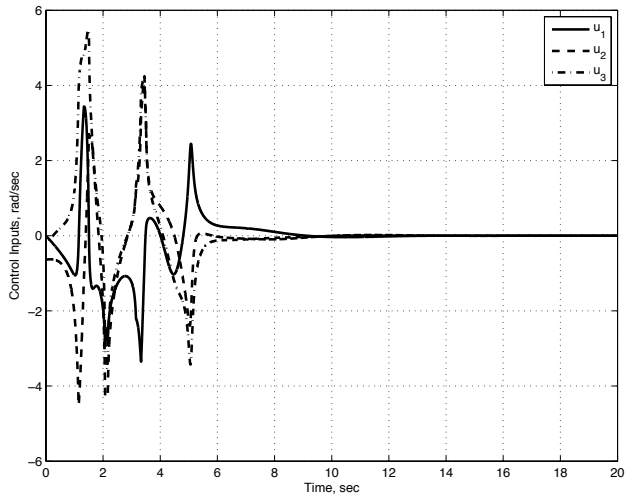

(d) Control input to the gimbals.

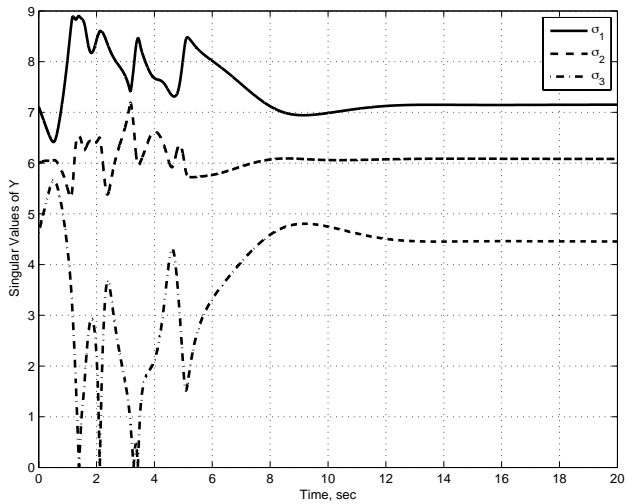

(f) Singular values of the matrix $Y$.
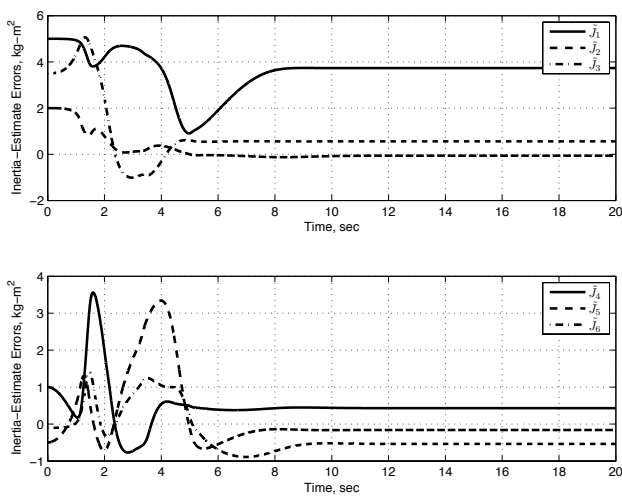

(g) Inertia-estimate errors.

Figure 5: Rest-to-Rest maneuver with CMGs using control law (58). 


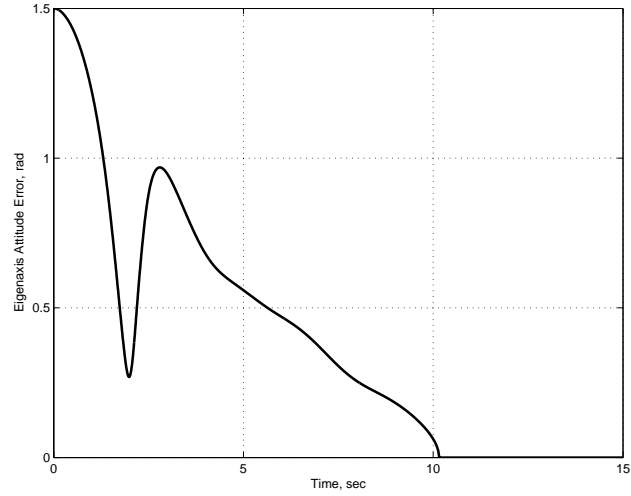

(a) Eigenaxis attitude error.

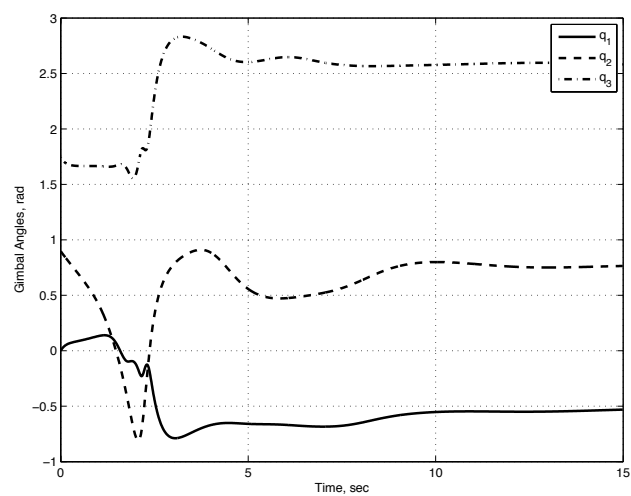

(c) Gimbal Angles.

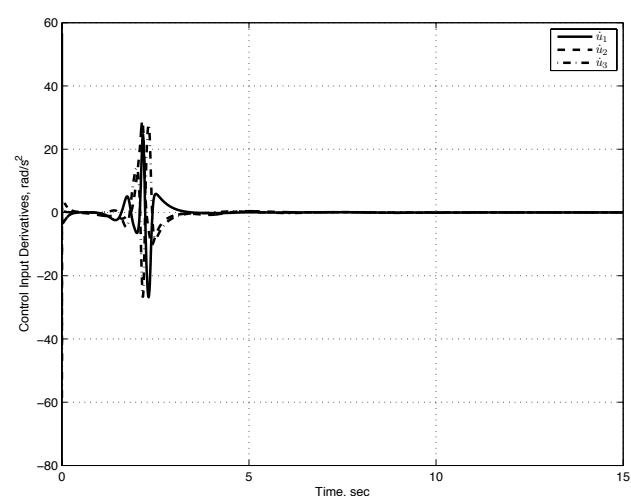

(e) Control input derivatives of the gimbals.

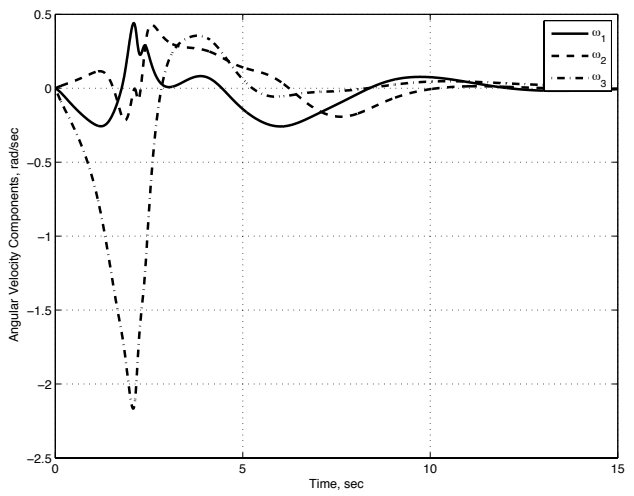

(b) Spacecraft angular-velocity components.

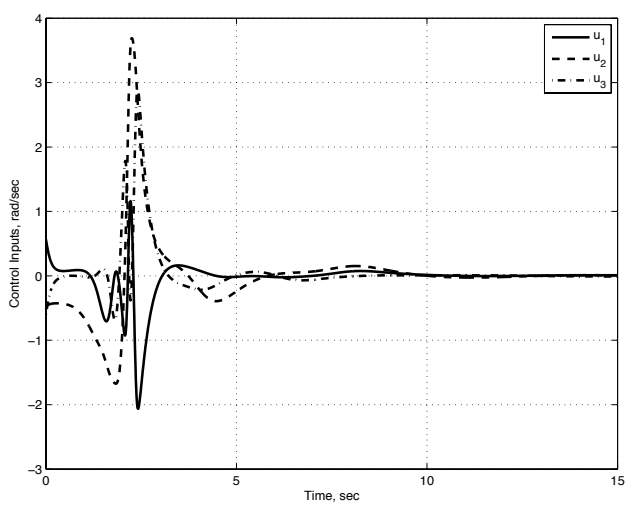

(d) Control input to the gimbals.

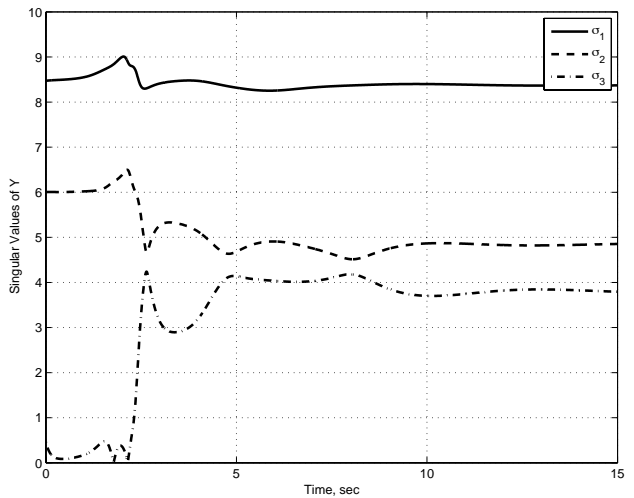

(f) Singular values of the matrix $Y$.
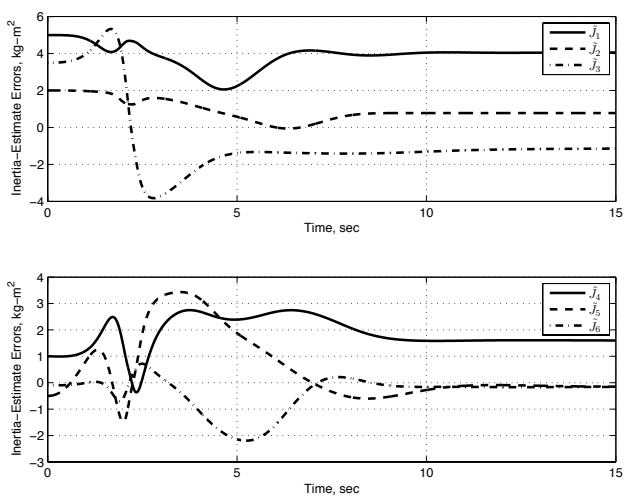

(g) Inertia-estimate errors.

Figure 6: Rest-to-Rest maneuver with CMGs using control law (63). 


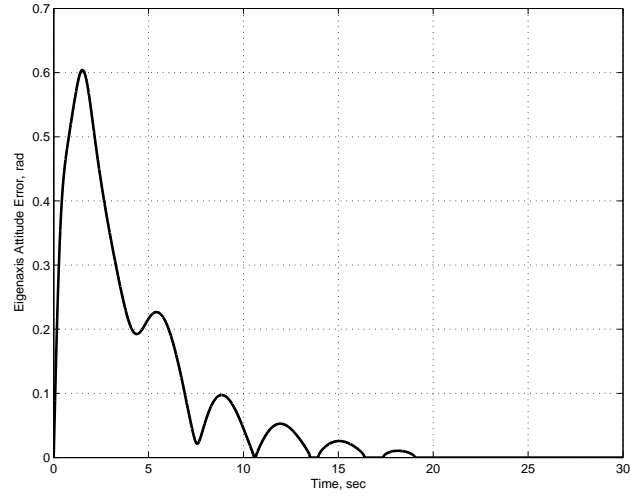

(a) Eigenaxis attitude error.

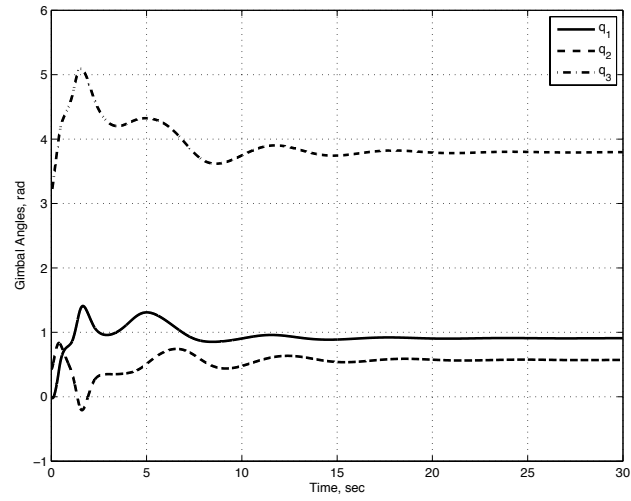

(c) Gimbal Angles.

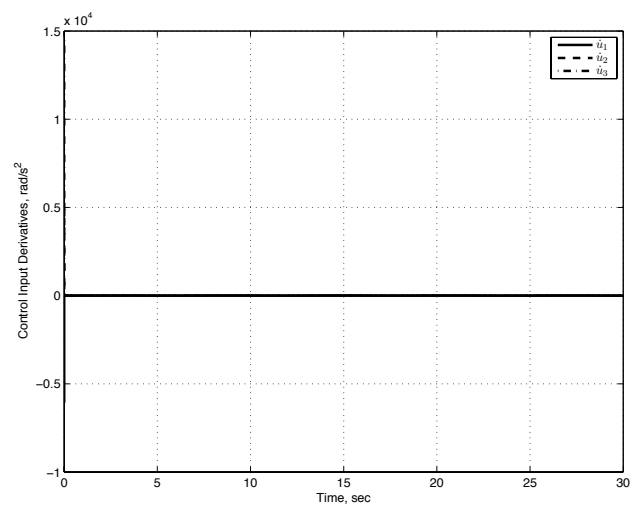

(e) Control input derivatives of the gimbals.



(b) Spacecraft angular-velocity components.

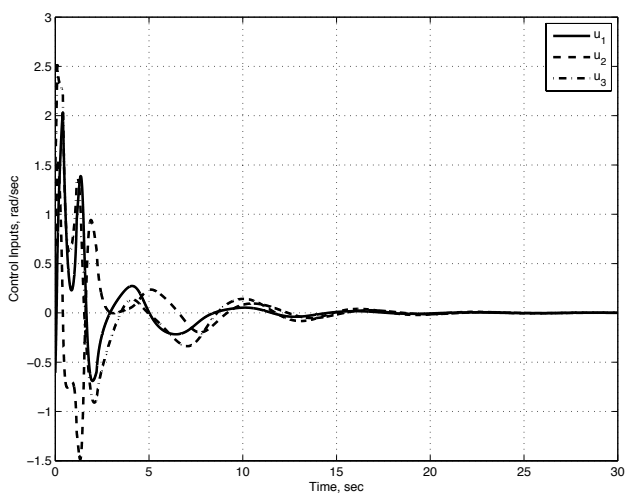

(d) Control input to the gimbals.

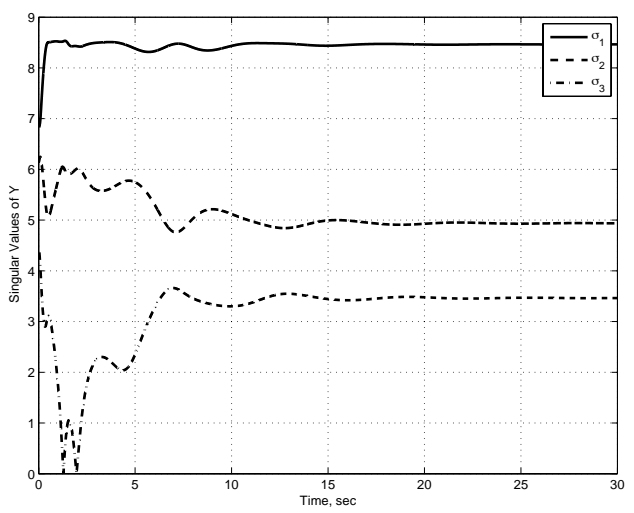

(f) Singular values of the matrix $Y$.


(g) Inertia-estimate errors.

Figure 7: Motion-to-Rest maneuver with CMGs using control law (58).

24 of 26 


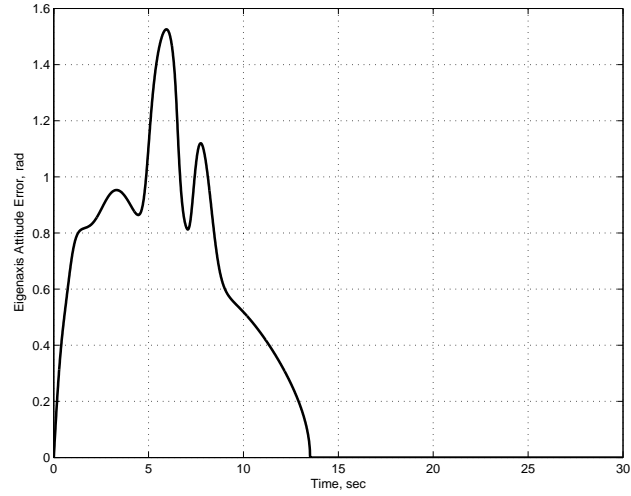

(a) Eigenaxis attitude error.

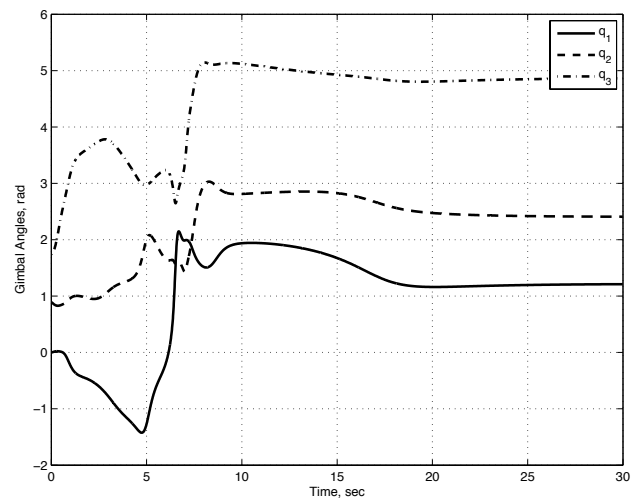

(c) Gimbal Angles.

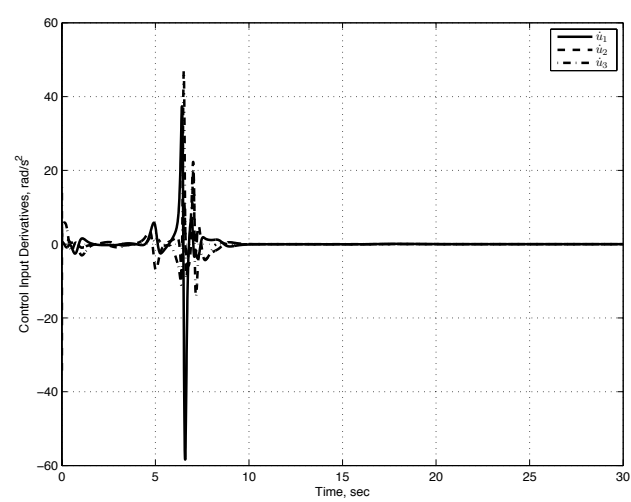

(e) Control input derivatives of the gimbals.

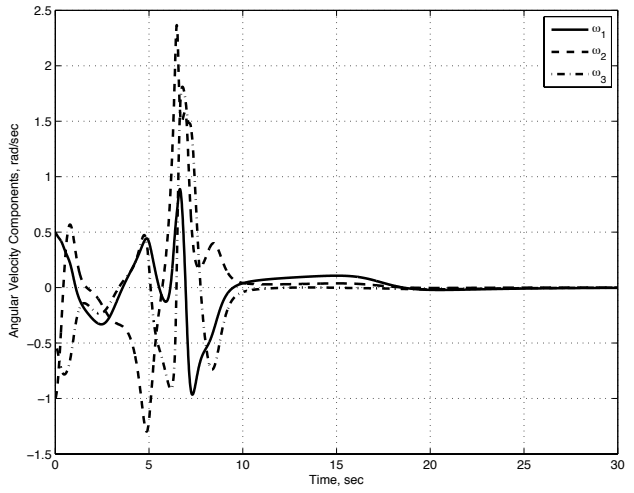

(b) Spacecraft angular-velocity components.

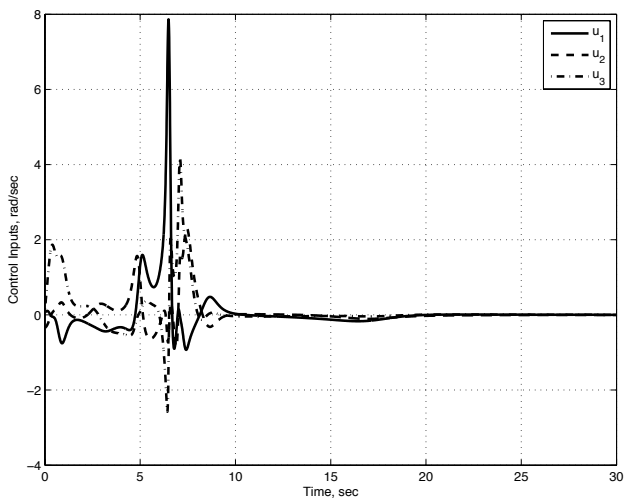

(d) Control input to the gimbals.

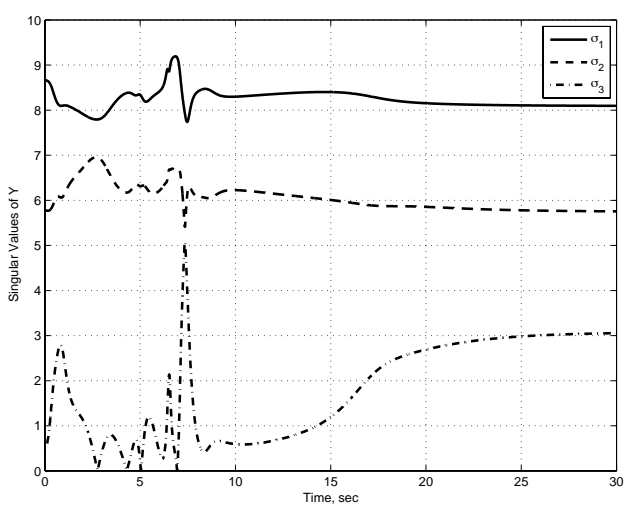

(f) Singular values of the matrix $Y$.
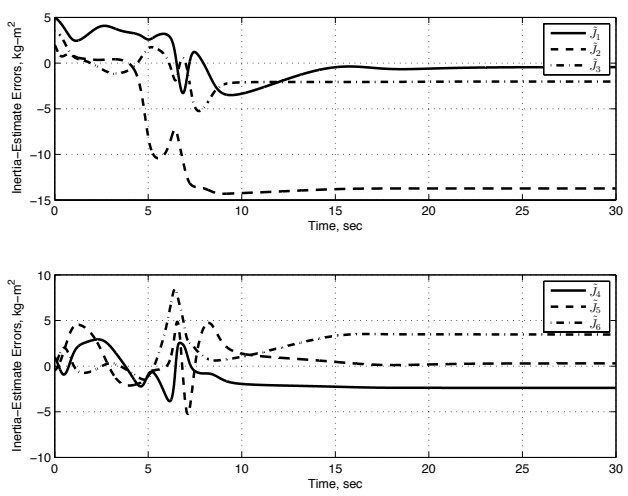

(g) Inertia-estimate errors.

Figure 8: Motion-to-Rest maneuver with CMGs using control law (63). 




(a) Eigenaxis attitude error.

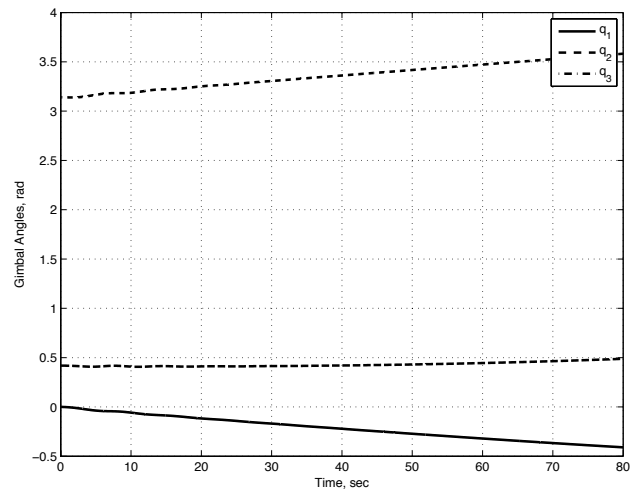

(c) Gimbal Angles.



(e) Control input derivatives of the gimbals.

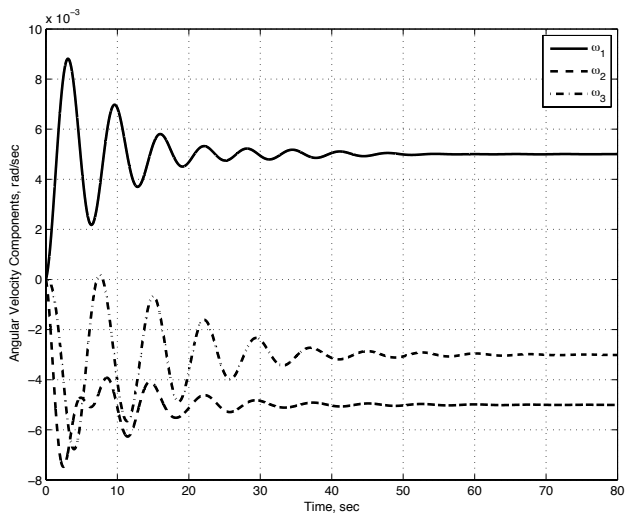

(b) Spacecraft angular-velocity components.

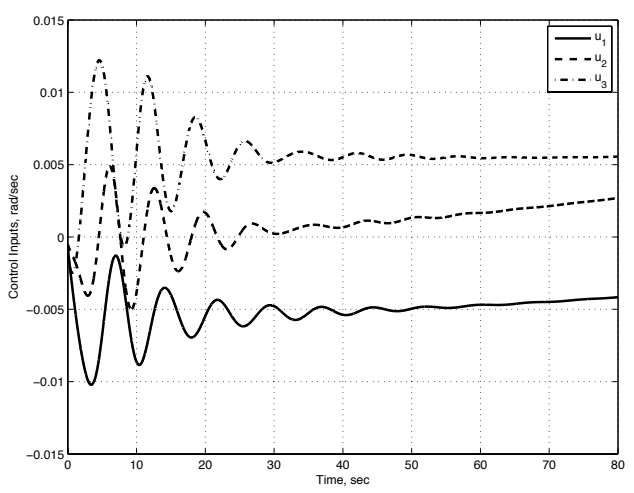

(d) Control input to the gimbals.

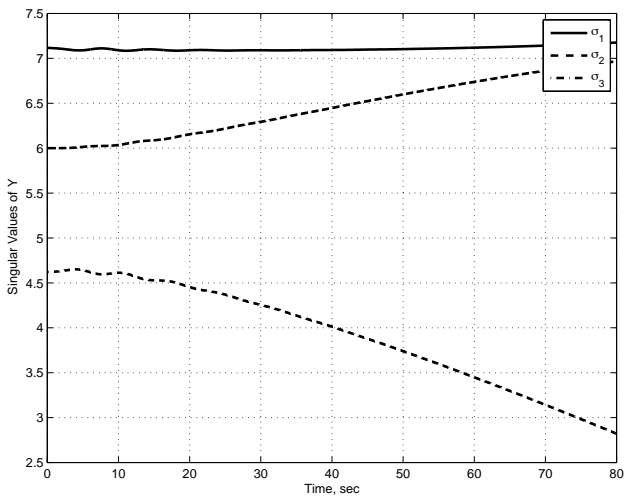

(f) Singular values of the matrix $Y$.
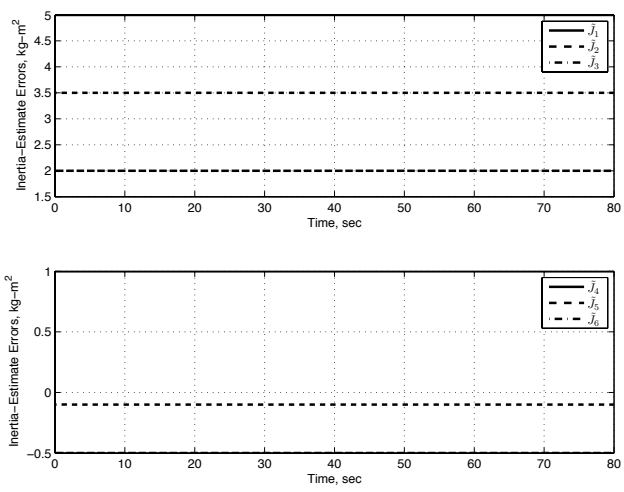

(g) Inertia-estimate errors.

Figure 9: Spin maneuver with CMGs using control law (63). 